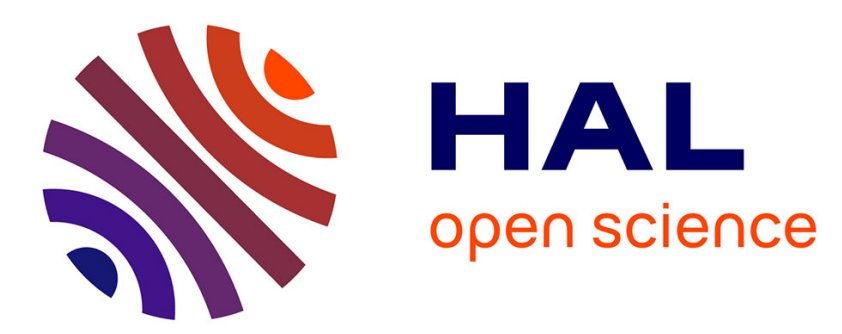

\title{
Dispositif de microélectrophorèse utilisant un vélocimètre optique original à réseau de transmission : aspects théoriques et méthodologiques
}

\author{
E. Delatour-Hanss
}

\section{- To cite this version:}

E. Delatour-Hanss. Dispositif de microélectrophorèse utilisant un vélocimètre optique original à réseau de transmission: aspects théoriques et méthodologiques. Revue de Physique Appliquée, 1989, 24 (9), pp.951-971. 10.1051/rphysap:01989002409095100 . jpa-00246131

\section{HAL Id: jpa-00246131 https://hal.science/jpa-00246131}

Submitted on 1 Jan 1989

HAL is a multi-disciplinary open access archive for the deposit and dissemination of scientific research documents, whether they are published or not. The documents may come from teaching and research institutions in France or abroad, or from public or private research centers.
L'archive ouverte pluridisciplinaire HAL, est destinée au dépôt et à la diffusion de documents scientifiques de niveau recherche, publiés ou non, émanant des établissements d'enseignement et de recherche français ou étrangers, des laboratoires publics ou privés. 


\title{
Dispositif de microélectrophorèse utilisant un vélocimètre optique original à réseau de transmission : aspects théoriques et méthodologiques
}

\author{
E. Delatour-Hanss \\ Laboratoire de Biophysique, U.F.R. de Médecine, 74 rue Marcel Cachin, 93012 Bobigny Cedex, France
}

(Reçu le 20 février 1989, accepté le 8 juin 1989)

\begin{abstract}
Résumé. - Nous avons étudié et réalisé un nouveau dispositif de microélectrophorèse, utilisant un vélocimètre optique à réseau de transmission. L'image en fond noir d'une suspension de particules est formée sur un réseau de transmission (fentes verticales alternativement transparentes et opaques); si les particules diffusantes se déplacent perpendiculairement aux fentes du réseau, la lumière transmise est modulée en fonction du temps; l'analyse spectrale de cette modulation permet de mesurer la vitesse des particules, connaissant le pas du réseau. Le principe de la vélocimétrie à réseau de transmission est rappelé et développé, de façon à pouvoir relier les résultats expérimentaux (spectres de puissance) à la distribution des vitesses des particules observées. Le dispositif expérimental mis au point (système optique spécialement adapté, cuve d'électrophorèse pratique avec plans stationnaires bien définis, système de traitement de signal performant et souple d'emploi), permet d'obtenir rapidement des spectres de mobilité électrophorétique de particules ou cellules en suspension. Nous présentons quelques études réalisées sur des suspensions diluées de globules rouges humains, qui illustrent la précision du dispositif, et sa grande résolution en vitesse.
\end{abstract}

\begin{abstract}
A new microelectrophoresis device has been studied and designed, which uses a transmission grating velocimeter. The dark-field image of suspended particles is focused on a transmission grating (transparent and opaque vertical slits); if the particles move in a direction perpendicular to the grating slits, the transmitted light intensity is time-modulated : the spectral analysis of this signal allows the determination of the particles velocity, knowing the grating spacing. The principle of the transmission grating velocimeter is recalled and developed, in order to deduce the velocity distribution of the observed particles from the experimental results (power spectra). The described apparatus (specially designed optics, rectangular electrophoretic chamber with well-defined stationary planes, powerful signal processing) gives rise to highresolution electrophoretic mobility spectra, in a very short time. Some studies on diluted human red blood cells suspensions are presented.
\end{abstract}

\section{Introduction.}

Les propriétés électriques de surface de particules colloïdales en suspension (charge ou potentiel) donnent des renseignements précieux aussi bien sur leur structure que sur leur possibilité d'interaction mutuelle ou avec d'autres types de surfaces; la connaissance de ces propriétés est fondamentale dans plusieurs domaines : physico-chimie, biologie, médecine ou industrie. Une des méthodes d'approche des propriétés électriques consiste à étudier la migration des particules chargées dans un champ électrique ; cette technique (électrophorèse) a plusieurs variantes, analytiques ou préparatives, largement utilisées en biologie et en chimie colloïdale : micro-électrophorèse (pour les particules visibles au microscope optique), électrophorèse libre (ou de frontière), électrophorèse de zone (ou sur support).

L'électrophorèse en phase liquide est une méthode physique particulièrement bien adaptée à l'étude des cellules vivantes car elle ne produit pas d'altération ou de destruction de l'organisation cellulaire (contrairement aux techniques biochimiques). De plus, elle permet de suivre l'évolution de toute réaction chimique, enzymatique, immunologique ou virale qui change le nombre ou la structure des groupements chargés surfaciques : cette information est très utile, car la région périphérique ou enveloppe cellulaire (membrane) est le siège de phénomènes biologiques importants intervenant dans différents domaines tels que l'enzymologie, l'immunologie, la virologie ou la pathologie. 
Pour déterminer la mobilité électrique de particules ou cellules en suspension, il existe deux types de techniques, basées soit sur une mesure de déplacement (méthode microscopique), soit sur une mesure de vitesse (méthodes vélocimétriques). La première consiste à mesurer le déplacement des particules sous l'action d'un champ électrique constant donné, pendant un intervalle de temps connu (ou mesure du temps pour un déplacement connu). Cette méthode classique [1-5], basée sur l'observation microscopique (d'où le nom de microélectrophorèse), est longue, fastidieuse, et subjective puisque les particules à mesurer sont choisies par l'opérateur.

Des modifications ont été apportées à la méthode microscopique, notamment pour éviter l'intervention directe de l'expérimentateur : photographie des trajectoires des particules, soumises à un champ électrique alternatif et emploi de techniques numériques [6-9], ou adaptation d'un ensemble vidéo et traitement d'image [10-12]. Ces méthodes ne sont toutefois pas très pratiques lorsque l'on fait des mesures répétitives pour déterminer des spectres de mobilité. Ceci explique l'emploi fréquent des méthodes vélocimétriques, plus rapides, en particulier celles basées sur l'effet Doppler, c'est-à-dire le changement de fréquence que subit la lumière lorsqu'elle est diffusée par des particules en mouvement. Cette technique, qui nécessite l'emploi de sources monochromatiques très intenses (donc de lasers), fut employée pour la première fois par Yeh et Cummins en 1964 [13]. La vélocimétrie Dopplerlaser, dont il existe différentes variantes [14], a été utilisée sous champ électrique en veine liquide initialement par Ware et Flygare [15, 16], et Uzgiris $[17,18]$; depuis de nombreux groupes ont développé et adapté cette méthode en fonction des différents types d'échantillons étudiés [19-27]. Une mise au point sur la méthode et ses applications a été publiée par Uzgiris [28, 29], et Ware et Haas [30].

L'électrophorèse par vélocimétrie Doppler est une méthode rapide et précise, permettant d'obtenir des spectres de mobilité sur un grand nombre de particules. Mais la réalisation et la mise en œuvre de cette méthode ne sont pas toujours faciles, et certains inconvénients (comme le phénomène d'électroosmose) rendent l'interprétation des spectres délicate. C'est pourquoi nous avons étudié et réalisé un nouveau dispositif de microélectrophorèse, utilisant une source lumineuse conventionnelle et une méthode vélocimétrique à réseau.

D'une façon générale, la vélocimétrie à réseau peut être basée sur un des procédés optiques suivants :

- on forme dans la suspension une succession périodique de zones éclairées (réseau d'illumination dans la direction $x$ ) ; les particules se déplacent selon la direction $x$, la lumière globale issue de la zone où est formé le réseau est modulée dans le temps [3133] ;

- on forme l'image de la suspension sur un réseau de transmission (périodique selon la direction $x$ ) ; si les particules se déplacent selon la direction $x$, la lumière transmise est modulée [34].

Nous rappelons et développons le principe de la méthode optique utilisée (vélocimétrie à réseau de transmission), afin de pouvoir déterminer la distribution des vitesses des particules, à partir de l'analyse spectrale de l'intensité lumineuse. Puis nous décrivons le dispositif expérimental mis au point : le système optique, la cuve d'électrophorèse et le système d'acquisition et de traitement du signal, ainsi que le protocole de mesure utilisé lors de l'étude électrophorétique d'une suspension. Nous rapportons ensuite quelques études avec ou sans champ électrique, réalisées afin d'étalonner le montage vélocimétrique et d'en vérifier le bon fonctionnement, en particulier en ce qui concerne le caractère local des mesures, et la résolution en vitesse. Enfin, nous présentons un exemple d'étude électrophorétique, réalisée sur des suspensions diluées de globules rouges humains.

\section{Principe du vélocimètre.}

Le vélocimètre que nous décrivons [35-37] est basé sur la modulation temporelle de la lumière transmise par un réseau, lorsqu'il est éclairé par des particules diffusantes en mouvement. Différents procédés peuvent être choisis pour obtenir l'image de la suspension sur le réseau : nous avons adopté l'image en fond noir de façon à obtenir le meilleur contraste possible.

2.1 PRINCIPE GÉNÉRAL. - Le réseau de transmission (Fig. 1) est caractérisé par la fonction de transmission $T(x, y)$, égale à 0 à l'extérieur des fentes transparentes, et égale à 1 à l'intérieur des fentes. En mode « fond noir », la lumière diffusée par chaque particule est focalisée sur le réseau et crée une image brillante. L'ensemble de ces sources lumineuses secondaires est décrit par une fonction d'éclairement $E(x, y)$. Le flux lumineux total transmis par le réseau est donc:

$$
\Phi=\iint_{-\infty}^{+\infty} T(x, y) E(x, y) \mathrm{d} x \mathrm{~d} y .
$$

Si la suspension est immobile, le flux total transmis est constant. Si les images des particules se déplacent toutes à la même vitesse $V$ selon la direction $x$, le flux transmis est modulé suivant la relation:

$$
\begin{aligned}
\Phi(t) & =\iint_{0} T(x) E(x-V t, y) \mathrm{d} x \mathrm{~d} y \\
\text { ou } \quad \Phi(t) & =\int_{-h / 2}^{+h / 2} T(V t) \otimes E(-V t, y) \mathrm{d} y .
\end{aligned}
$$




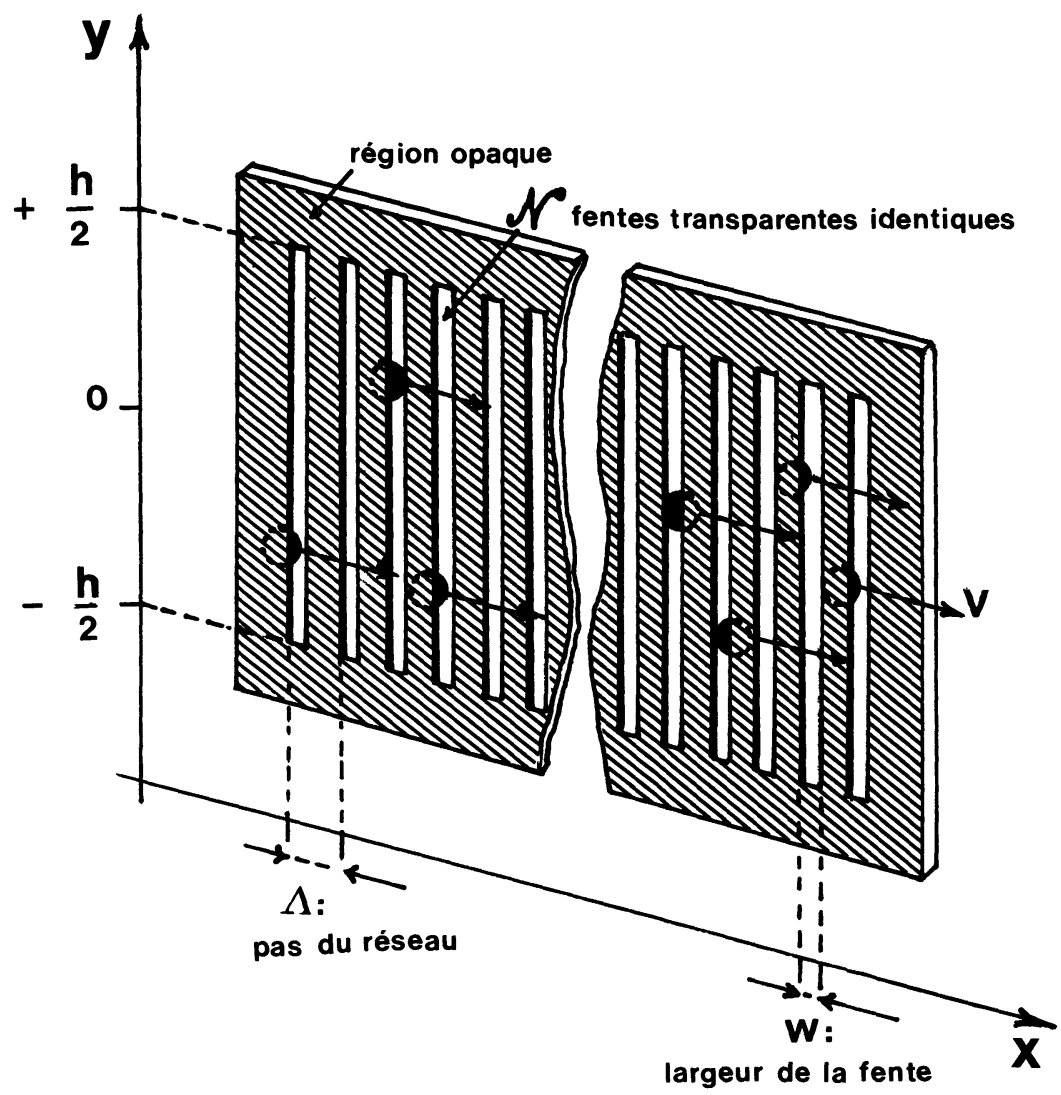

Fig. 1. - Caractéristiques du réseau de transmission.

[Specifications of the transmission grating.]

Dans cette expression $\Phi(t)$ est la convolution $(\otimes)$ des deux fonctions $T(x)$ et $E(x)$, intégrée sur la longueur d'une fente transparente; elle dépend de la vitesse des particules, $V$. Après transformation de Fourier de $\Phi(t)$, on obtient le spectre en fréquences $S(F)$ donné par la relation suivante :

$$
S(F)=V \int_{-h / 2}^{+h / 2} \mathbf{F} T(V t) \cdot \mathbf{F} E(-V t, y) \mathrm{d} y
$$

où $\mathbf{F} T(V t)$ et $\mathbf{F} E(-V t, y)$ sont les transformées de Fourier de $T(V t)$ et de $E(-V t, y)$.

Etant donné la forme mathématique de la fonction $T(V t)$ (la fonction $T(x)$ est égale à 1 à l'intérieur des $N$ fentes, et égale à 0 à l'extérieur), un calcul simple de transformations de Fourier [38] donne :

$$
\begin{aligned}
S(F)=\frac{N \Lambda}{m} \sum_{p} \frac{\sin \frac{\pi p}{m}}{\frac{\pi p}{m}} & \frac{\sin \pi\left(F-\frac{p V}{\Lambda}\right) \frac{N \Lambda}{V}}{\pi\left(F-\frac{p V}{\Lambda}\right) \frac{N \Lambda}{V}} \times \\
& \times \int_{-h / 2}^{+h / 2} \mathbf{F} E(-V t, y) \mathrm{d} y
\end{aligned}
$$

$p$ étant un nombre entier (de $-\infty$ à $+\infty)$, et $m=\Lambda / w$.

Cette expression montre que le spectre en fréquences est composé d'une suite d'harmoniques de la fréquence fondamentale $F_{1}=V / \Lambda$, d'amplitude variable (facteur $(\sin \pi p / m) / \pi p / m)$ et dépendant de l'éclairement du réseau (par l'intermédiaire de l'intégrale $\left.\int \mathrm{F} E(-V t, y) \mathrm{d} y\right)$. La mesure de $F_{1}$ permet donc de connaître la vitesse $V$.

L'intégrale intervenant dans cette équation dépend d'une part de la répartition statistique des images des particules sur le réseau, d'autre part de la répartition de l'intensité lumineuse à l'intérieur de chaque image (elle-même fonction de la forme, des dimensions, et de l'orientation de la particule diffusante). De plus, l'équation (1) n'est valable que pour des particules se déplaçant à vitesse uniforme. On conçoit la complexité du problème en général : en effet, les caractéristiques géométriques (forme, dimensions, orientation) peuvent varier d'une particule à l'autre, et les particules ont une certaine distribution de vitesse; enfin, ces distributions ne sont pas nécessairement indépendantes (par exemple la vitesse peut influencer l'orientation des particules).

Nous allons résoudre l'équation (1) en procédant par étapes, et en faisant des hypothèses simplificatrices sur la fonction d'éclairement. Nous allons d'abord examiner ce que devient cette équation dans le cas d'une seule particule diffusante, puis pour un ensemble de particules identiques et de même 
vitesse, avant de généraliser à des suspensions de particules hétérogènes dans leurs caractéristiques géométriques, et de vitesse variable.

2.2 EXPRESSION DE $S_{0}(F)$, SPECTRE EN FRÉQUENCES POUR UNE SEULE PARTICULE. - Le spectre en fréquences $S_{0}(F)$ résultant du passage d'une particule-image à travers le réseau est donné par l'équation (1), où $E(x, y)$ est l'éclairement dû à la diffusion par la particule. Dans le domaine qui nous intéresse (colloïdes et cellules), où les particules ont des dimensions plus grandes que la longueur d'onde de la lumière utilisée, la théorie de la diffusion est difficile, et n'est résolue complètement que pour des sphères (théorie de Mie [39]). Cependant pour notre problème, il n'est pas nécessaire de connaître la répartition exacte de l'intensité lumineuse dans l'image de diffraction de la particule, car elle influence peu la forme du spectre; on peut le montrer facilement [37], en donnant à $E(x, y)$ quelques formes analytiques simples (par exemple éclairement uniforme $(=C$ ) sur une surface carrée, circulaire ou elliptique). Le spectre $S_{0}(F)$ se met alors sous la forme :

$$
\begin{aligned}
& S_{0}(F)=\varphi \frac{N \Lambda}{m V} f(F) \times \\
& \times \sum_{p}\left(\frac{\sin \frac{\pi p}{m}}{\frac{\pi p}{m}}\right)\left(\frac{\sin \pi\left(F-\frac{p V}{\Lambda}\right) \frac{N \Lambda}{V}}{\pi\left(F-\frac{p V}{\Lambda}\right) \frac{N \Lambda}{V}}\right)
\end{aligned}
$$

où $\varphi$ est le flux lumineux incident sur le réseau, dû à la diffusion par la particule $(\varphi=C s$, où $s$ est l'aire de l'image de la particule sur le réseau) et $f(F)$ est une fonction de $F$, égale à 1 pour $F=0$, dépendant de la forme et des dimensions de l'image, variant lentement par rapport au facteur

$$
\frac{\sin \pi\left(F-\frac{p V}{\Lambda}\right) \frac{N \Lambda}{V}}{\pi\left(F-\frac{p V}{\Lambda}\right) \frac{N \Lambda}{V}}
$$

Par exemple, pour des images de formes carrée (côté $a, \varphi=C a^{2}$ ) et circulaire (rayon $a$, $\left.\varphi=C \pi a^{2}\right)$, la fonction $f(F)$ est respectivement égale à $[\sin (\pi F a / V)] /(\pi F a / V)$ et $\left[2 J_{1}(2 \pi F a / V)\right] /(2 \pi F a / V), J_{1}$ étant la fonction de Bessel d'ordre 1. Remarquons que si les dimensions de l'image sont très petites, $f(F)=1$ indépendamment de la forme de l'image.

L'expression (2) est une suite d'harmoniques, chacun centré autour d'une valeur de la fréquence $F=p V / \Lambda$, et multiplié par le facteur de forme $f(F)$ et le facteur constant $(\sin \pi p / m) /(\pi p / m)$.

En élevant l'expression (2) au carré, on obtient le spectre d'énergie $\left|S_{0}(F)\right|^{2}$. Si $N \gg 1$ (ce qui est toujours le cas puisque les réseaux dont nous dispo- sons ont au minimum 100 fentes), les différents termes de la somme $\sum_{p}$ n'interfèrent pas car la largeur de chaque pic $(\approx 2 V / N \Lambda)$ est beaucoup plus petite que la distance entre pics $(=V / \Lambda)$, si bien que les termes rectangles dans le carré de la somme sont nuls, et le spectre d'énergie peut s'écrire :

$$
\begin{aligned}
& \left|S_{0}(F)\right|^{2}=\left(\varphi \frac{N \Lambda}{m V}\right)^{2} f^{2}(F) \times \\
& \times \sum_{p}\left(\frac{\sin \frac{\pi p}{m}}{\frac{\pi p}{m}}\right)^{2}\left(\frac{\sin \pi\left(F-\frac{p V}{\Lambda}\right) \frac{N \Lambda}{V}}{\pi\left(F-\frac{p V}{\Lambda}\right) \frac{N \Lambda}{V}}\right)^{2} .
\end{aligned}
$$

La figure 2 donne la représentation graphique du spectre d'énergie $\left|S_{0}(F)\right|^{2}$, pour $F \geqslant 0$, dans le cas d'une image de forme circulaire de rayon $a$. Nous remarquons que les facteurs de modulation $\left[2 J_{1}(2 \pi F a / V)\right]^{2} /(2 \pi F a / V)^{2}$ et $(\sin \pi p / m)^{2} /$ $(\pi p / m)^{2}$, dont les premiers zéros sont respectivement $F=1,22 V / 2 a$ et $p=m$ (ou $F=m V / \Lambda)$, varient lentement par rapport à chaque pic.

Chaque harmonique se compose d'un pic central (s'annulant pour $F=p V / \Lambda \pm V / N \Lambda$ ), et de lobes latéraux décroissant de part et d'autre (Fig. 3). La largeur à la base du pic central est $2 V / N \Lambda$. A mihauteur, la largeur est $\Delta F \approx(0,885 / N) V / \Lambda$ [37], ou en valeur relative par rapport à la fréquence fondamentale : $\Delta F / F_{1}=0,885 / N<1 \%$ puisque $N>100$.

Ces exemples sont bien sûr très schématiques : dans la réalité, l'éclairement $E(x, y)$ n'est pas uniforme sur la surface de l'image, et la forme de celleci est plutôt voisine d'un anneau plus ou moins large, circulaire ou elliptique suivant l'orientation de la particule diffusante par rapport à la lumière incidente. Mais on peut toujours mettre le spectre en fréquences $S_{0}(F)$ d'une particule sous la forme (2), en posant :

$$
V \int_{-h / 2}^{+h / 2} \mathbf{F} E(-V t, y) \mathrm{d} y=\varphi f(F)
$$

$\varphi$ étant le flux incident dû à la particule et $f(F)$ une fonction de la fréquence, égale à 1 pour $F=0$, dépendant de l'image de diffraction de la particule, de forme analytique inconnue, mais qui tend vers 1 si les dimensions de l'image deviennent très petites.

\subsection{SPECTRE EN FRÉQUENCES POUR UN ENSEMBLE} DE PARTICULES IDENTIQUES ET DE MEME VITESSE. - Supposons toutes les particules identiques (mêmes caractéristiques géométriques) et de même vitesse ( $V$ au niveau du réseau), et en concentration assez faible pour que les différentes images n'empiètent pas les unes sur les autres. Si $\Phi_{0}(t)$ est le flux 


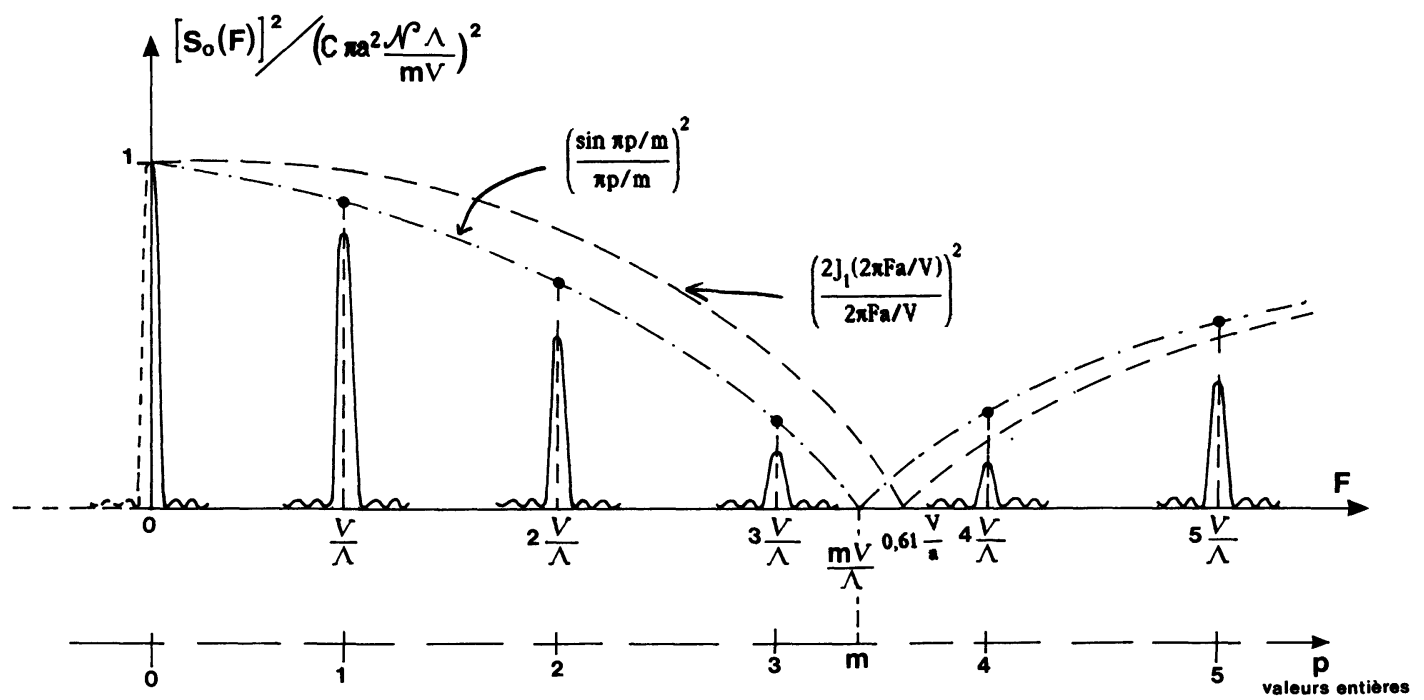

Fig. 2. - Représentation graphique de $\left[S_{0}(F)\right]^{2}$, spectre d'énergie pour une seule particule, dont l'image est de forme circulaire (rayon a). Dans cet exemple, on a $3<m<4$, et $2 a<1,22 \Lambda / m$.

[Graph of $\left[S_{0}(F)\right]^{2}$, energy spectrum for one single particle (circular image of radius $a$ ). In this example : $3<m<4$, and $2 a<1.22 \Lambda / m$.]

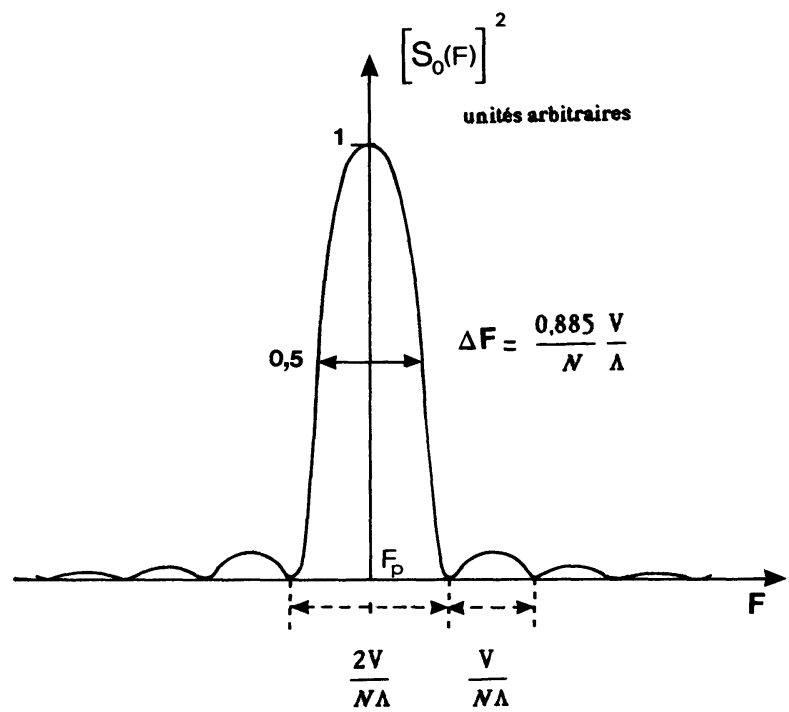

Fig. 3. - Représentation graphique de l'harmonique $p$ du spectre $\left[S_{0}(F)\right]^{2}$, centré sur $F_{p}=p V / \Lambda$.

[Graph of harmonic $p$ of the spectrum $\left[S_{0}(F)\right]^{2}$, centered at $F_{p}=p V / \Lambda$.]

transmis par le réseau, dû à la diffusion par une seule particule, le flux total transmis sera alors la somme des flux individuels :

$$
\Phi(t)=\sum_{i=-\infty}^{+\infty} \Phi_{0}\left(t+\theta_{i}\right) .
$$

$\Phi(t)$ peut donc être considérée comme la répétition à intervalles aléatoires $\theta_{i}$ de la fonction de carré intégrable $\Phi_{0}(t)$; c'est une fonction aléatoire stationnaire que l'on peut classer dans la catégorie
« shot noise », avec en moyenne $\alpha$ répétitions de la fonction $\Phi_{0}(t)$ par seconde $(\alpha=$ Cte $)$. Si $N_{\mathrm{s}}$ est le nombre moyen de particules-images par unité de surface du réseau de hauteur $h$, il y aura en moyenne $N_{\mathrm{s}} h V$ particules traversant une abscisse $x$ quelconque par unité de temps, d'où : $\alpha=N_{\mathrm{s}} h V$. En appelant $S_{0}(F)$ la transformée de Fourier de la fonction intégrable $\Phi_{0}(t)$, et $\langle\Phi(t)\rangle$ la valeur moyenne du flux total transmis, le spectre de puissance de la fonction shot noise $\Phi(t)$ s'écrit [37] :

$$
P(F)=N_{\mathrm{s}} h V\left|S_{0}(F)\right|^{2}+\langle\Phi(t)\rangle^{2} \delta(F)
$$

avec

$$
\langle\Phi(t)\rangle=N_{\mathrm{s}} h V \int_{-\infty}^{+\infty} \Phi_{0}(t) \mathrm{d} t=N_{\mathrm{s}} h N \Lambda \varphi / m .
$$

La variance et la fonction d'auto-corrélation de la fonction $\Phi(t)$ sont respectivement :

$\sigma_{\Phi}^{2}=\left\langle\Phi^{2}(t)\right\rangle-\langle\Phi(t)\rangle^{2}=N_{\mathrm{s}} h V \int_{-\infty}^{+\infty} \Phi_{0}^{2}(t) \mathrm{d} t$

et

$$
\begin{aligned}
c(t)=N_{\mathrm{s}} h V \int_{-\infty}^{+\infty} \Phi_{0}\left(t_{1}\right) \Phi_{0}\left(t_{1}+t\right) \mathrm{d} t_{1} & \\
+ & +\langle\Phi(t)\rangle^{2} .
\end{aligned}
$$

Nous observons que le flux total transmis $\Phi(t)$, qui est un signal aléatoire stationnaire dont la valeur moyenne $\langle\Phi\rangle$ n'est pas nulle, a donc dans son spectre de puissance une fonction delta d'amplitude $\langle\Phi\rangle^{2}$ positionnée en $F=0$, superposée à un spectre 
de puissance distribué sur toutes les fréquences, caractéristique des fluctuations de $\Phi(t)$ par rapport à la moyenne.

Si nous donnons au spectre $S_{0}(F)$ la forme (2), le spectre de puissance s'écrira donc, avec toujours la condition $N \gg 1$ :

$$
\begin{aligned}
& P(F)=\frac{N_{s} h}{V}\left(\frac{\varphi N \Lambda}{m}\right)^{2} f^{2}(F) \sum_{p}\left(\frac{\sin \frac{\pi p}{m}}{\frac{\pi p}{m}}\right)^{2} \times \\
& \left(\frac{\sin \pi\left(F-\frac{p V}{\Lambda}\right) \frac{N \Lambda}{V}}{\pi\left(F-\frac{p V}{\Lambda}\right) \frac{N \Lambda}{V}}\right)^{2}+\langle\Phi(t)\rangle^{2} \delta(F)
\end{aligned}
$$

La figure 4, semblable à la figure 2, donne l'allure générale du graphe de $P(F)$ (Eq. (4)), pour $F>0$ (la fonction Dirac n'est pas représentée). Outre un pic à la fréquence $F=0$ (correspondant à $p=0$ ), qui ne permet pas de mesurer la vitesse $V$, on a un premier maximum à la fréquence fondamentale $F_{1}=V / \Lambda$, et une série d'harmoniques. La mesure de $F_{1}$ permet donc de connaître $V$.

Remarquons que la fonction $f(F)$ inconnue varie lentement par rapport à chaque pic parce que les dimensions de l'image d'une particule sont très petites par rapport à la longueur totale du réseau, $N \Lambda$.

Chaque harmonique a un profil analogue à celui de la figure 3 , avec une amplitude proportionnelle à $N_{\mathrm{s}}$ et inversement proportionnelle à la vitesse $V$ (Eq. (4)). La surface de chaque harmonique (pic central et lobes latéraux) est par contre indépendante de la vitesse, et proportionnelle à $N_{\mathrm{s}}$ [37].

Le coefficient de variation $\sigma^{2} /\langle\Phi\rangle^{2}$, qui indique le taux de modulation du signal, est inversement proportionnel au nombre moyen de particules-ima- ges sur la surface du réseau $\left(=N_{\mathrm{s}} h N \Lambda\right)$, et dépend de la forme et des dimensions des particules (par l'intermédiaire de $f^{2}$ ). Pour avoir un taux de modulation correct, c'est-à-dire qui permette de mesurer au moins le pic fondamental, il faut choisir un réseau de pas supérieur aux dimensions de l'image de la particule.

\subsection{SPECTRE EN FRÉQUENCES POUR UN ENSEMBLE} DE PARTICULES IDENTIQUES ET DE VITESSE VARIABLE. - Supposons que la suspension contienne des particules identiques mais de vitesse variable, avec une distribution de vitesse discontinue $V_{1}, V_{2}, \ldots$ $V_{n}$. Parmi les $N_{\mathrm{s}}$ particules-images par unité de surface du réseau (nombre moyen), soit $N_{s j}$ le nombre de celles qui ont une vitesse égale à $V_{j}\left(\sum_{j=1}^{n} N_{\mathrm{s} j}=N_{\mathrm{s}}\right)$

Le spectre de puissance $P(F)$ s'écrit alors, d'après l'équation (4), si $N \gg 1$ :

$$
\begin{aligned}
P(F) & =\sum_{j} \frac{N_{s j} h}{V_{j}}\left(\frac{\varphi N \Lambda}{m}\right)^{2} f_{j}^{2}(F) \sum_{p}\left(\frac{\sin \frac{\pi p}{m}}{\frac{\pi p}{m}}\right)^{2} \times \\
& \times\left(\frac{\sin \pi\left(F-\frac{p V_{j}}{\Lambda}\right) \frac{N \Lambda}{V_{j}}}{\pi\left(F-\frac{p V_{j}}{\Lambda}\right) \frac{N \Lambda}{V_{j}}}\right)^{2}+\langle\Phi\rangle^{2} \delta(F) .
\end{aligned}
$$

Ainsi, pour $F \neq 0$, le spectre de puissance du flux total transmis est la somme des spectres de puissance individuels $P_{j}(F)$ correspondant à chaque vitesse $V_{j}$, chaque spectre $P_{j}$ étant fait d'une suite d'harmoniques centrés sur les fréquences $F=p V_{j} / \Lambda$, identique à la figure 4 (en remplaçant $N_{\mathrm{s}}$ par $N_{\mathrm{sj}}$ et $\mathrm{V}$ par $\left.V_{j}\right)$.

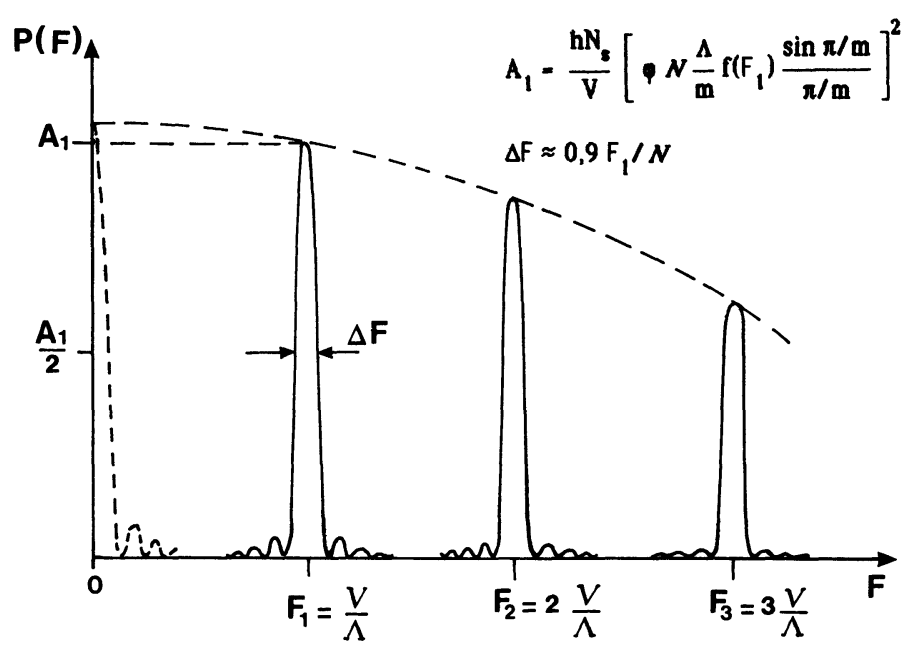

Fig. 4. - Spectre de puissance pour de nombreuses particules, identiques et de même vitesse $V(N \gg 1)$.

[Power spectrum for many identical particles, having the same velocity $V(N \gg 1)$.] 
Etant donné que les mesures de vitesse se font toujours à l'aide des fondamentales $p=1$ (d'amplitude plus grande), pour qu'il n'y ait pas de chevauchement avec d'autres harmoniques, il faut que l'harmonique $p=2$ de la vitesse minimum ait une fréquence plus grande que la fondamentale $p=1 \mathrm{de}$ la vitesse maximum, c'est-à-dire que $V_{\max }<2 V_{\min }$. $\mathrm{Si}$ la suspension à analyser a une distribution de vitesse plus large, on évite les interférences entre harmoniques 1 et 2 en annulant l'ensemble des harmoniques 2 (il suffit pour cela d'utiliser un réseau de $m=2$, dont les zones opaque et transparente ont même largeur) : dans ce cas, on peut mesurer sans ambiguité des vitesses comprises entre $V_{\min }$ et $3 V_{\min }$, à partir de l'équation suivante, pour $F>0$ :

$$
\begin{aligned}
P(F)=h\left(\frac{\varphi N \Lambda}{\pi}\right)^{2} \sum_{j} \frac{N_{s j}}{V_{j}} f_{j}^{2}(F) \times & \\
& \times\left(\frac{\sin \pi\left(F-\frac{V_{j}}{\Lambda}\right) \frac{N \Lambda}{V_{j}}}{\pi\left(F-\frac{V_{j}}{\Lambda}\right) \frac{N \Lambda}{V_{j}}}\right)^{2} .
\end{aligned}
$$

Résolution en vitesse de la méthode: si la suspension contient deux groupes de particules, de vitesses $V_{1}$ et $V_{2}$ proches l'une de l'autre, le spectre de puissance résultant est la somme des 2 spectres de puissance individuels (Fig. 5): $P(F)=P_{1}(F)+$ $P_{2}(F)$. On pourra distinguer les fondamentales $F_{1}$ et $F_{2}$, c'est-à-dire les vitesses $V_{1}$ et $V_{2}$ si $\Delta V / V$ est au moins égal à $1 / N$ (le maximum central d'un pic coïncidant avec le premier minimum nul de l'autre pic) ; $1 / N$ définit donc la résolution en vitesse théorique de la méthode, d'autant meilleure que le nombre de fentes du réseau est grand.
Dans le cas d'une distribution de vitesse continue $n_{\mathrm{s}}(v)$ (où $v_{\max }<3 v_{\min }$ ), l'équation (5), qui donne l'ensemble des fondamentales $p=1$ du spectre, valable pour $N \gg 1, m=2$ et $F>0$, s'écrira :

$$
\begin{aligned}
& P(F)=h\left(\frac{\varphi N \Lambda}{\pi} f\right)^{2} \int \frac{n_{\mathrm{s}}(v)}{v} \times \\
& \times\left(\frac{\sin \pi\left(F-\frac{v}{\Lambda}\right) \frac{N \Lambda}{v}}{\pi\left(F-\frac{v}{\Lambda}\right) \frac{N \Lambda}{v}}\right)^{2} \mathrm{~d} v .
\end{aligned}
$$

Cette équation montre que la mesure du spectre de puissance $P(F)$ n'entraîne pas automatiquement la connaissance de la distribution de vitesse $n_{\mathrm{s}}(v)$. En effet, l'intégrale de l'équation (6) ne se calcule facilement que si l'un des facteurs varie lentement par rapport à l'autre.

La figure 6 montre l'évolution qualitative du spectre $P(F)$ d'une suspension de particules (avec $N_{\mathrm{s}}=$ Cte), lorsque la largeur à mi-hauteur $\Delta v$ de la distribution de vitesse $n_{\mathrm{s}}(v)$ diminue, de part et d'autre de la vitesse moyenne $\bar{v}$. La surface du spectre étant constante, lorsque $\Delta v$ diminue l'amplitude du pic augmente et sa largeur à mi-hauteur $\Delta F$ diminue. Si la largeur relative $\Delta v / \bar{v}$ de la distribution de vitesse est grande devant la résolution théorique $1 / N$, alors $P(F)$ et $n_{\mathrm{s}}(v)$ ont même profil $\left(P(F)=K n_{\mathrm{s}}(F \Lambda)\right)$, avec $\Delta F=\Delta v / \Lambda$;" si $\Delta v / \bar{v}$ n'est pas grande devant $1 / N$, alors $P(F)$ diffère de $K n_{\mathrm{s}}(F \Lambda)$, et la largeur $\Delta F$ est plus grande que $\Delta v / \Lambda$, d'autant plus que $\Delta v$ est petite, jusqu'à devenir égale à la valeur $\bar{v} / N \Lambda$ lorsque $\Delta v=0$; dans ce dernier cas (vitesse $\bar{v}$ unique), l'amplitude du pic (Eq. (4)) est égale à $K N N_{\mathrm{s}} / \bar{v}$, et les lobes

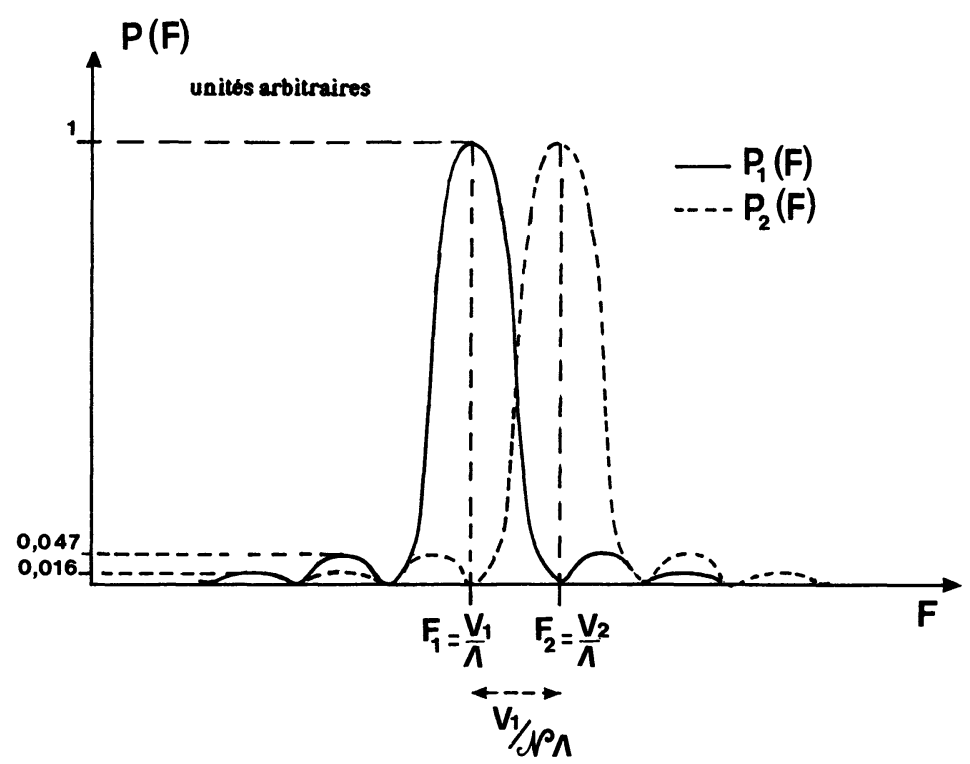

Fig. 5. - Spectre de puissance pour deux groupes de particules de vitesses voisines $V_{1}$ et $V_{2}$.

[Power spectrum for two types of particles, having near velocities $V_{1}$ and $V_{2}$.] 


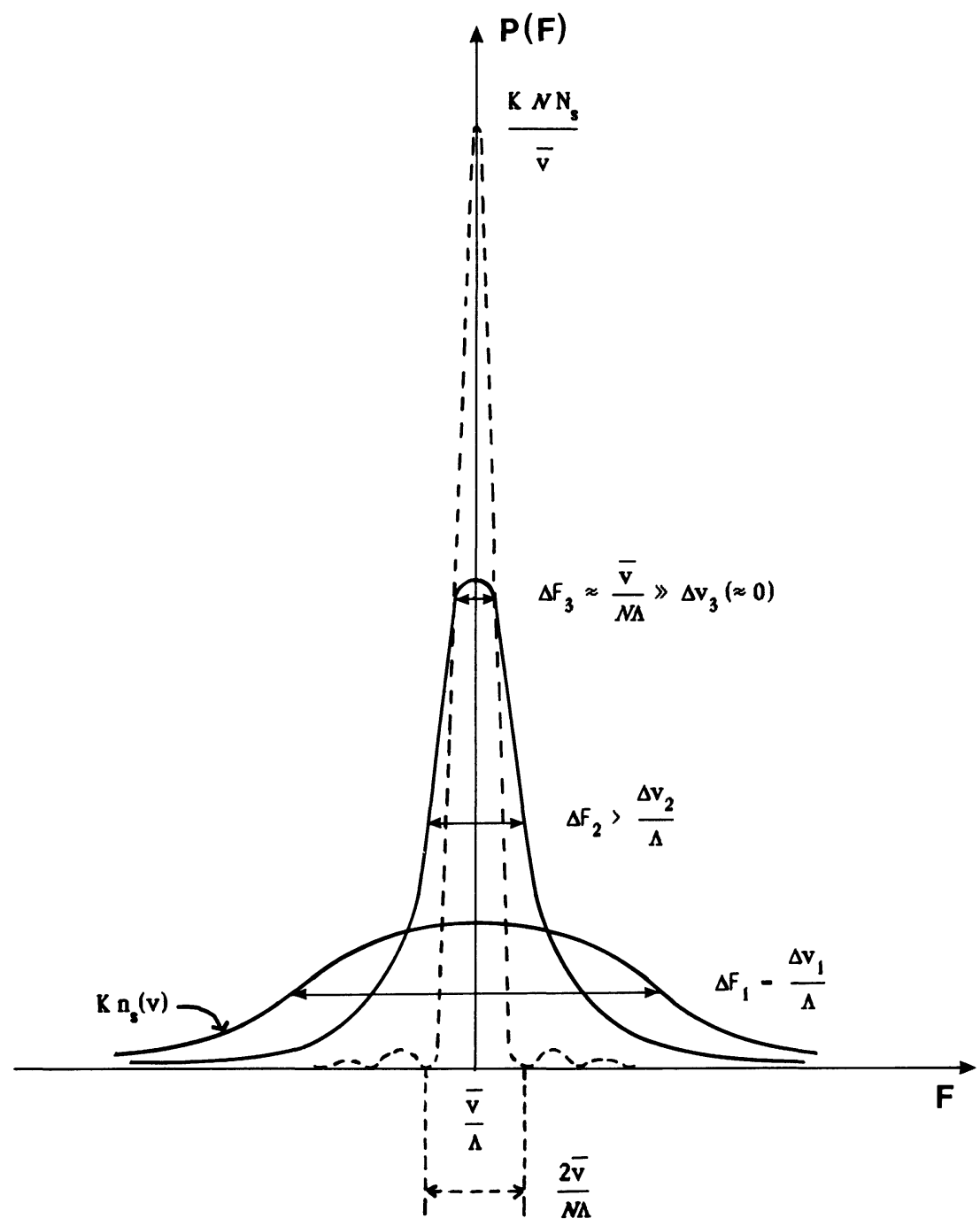

Fig. 6. - Evolution du spectre $P(F)$ d'une suspension de particules $\left(N_{\mathrm{s}}=\right.$ Cte ) lorsque la largeur à mi-hauteur $\Delta v$ de la distribution de vitesse $n_{\mathrm{s}}(v)$ diminue : courbe $1: \Delta v_{1} \gg v / N$, courbe $2: \Delta v_{2} \approx v / N$, courbe $3: \Delta v_{3} \leqslant v / N$ $\left(K=N h(\varphi \Lambda f / \pi)^{2}\right)$.

[Evolution of the spectrum $P(F)$ of a particles suspension (with constant $N_{\mathrm{s}}$ ), as the half-height width $\Delta v$ of the velocity distribution $n_{\mathrm{s}}(v)$ decreases : curve $1: \Delta v_{1} \gg v / N$, curve $2: \Delta v_{2} \approx v / N$, curve $3: \Delta v_{3} \ll v / N\left(K=N h(\varphi \Lambda f / \pi)^{2}\right)$.]

latéraux sont visibles (la courbe 3 obtenue est alors la « fonction d'appareil » du vélocimètre).

Pour analyser de manière quantitative le spectre obtenu avec une distribution de vitesse $n_{\mathrm{s}}(v)$ quelconque dont on ne connaît pas la largeur, on est obligé de faire des hypothèses sur la forme de la distribution, de calculer (par des méthodes numériques) le spectre théorique qui en résulte (Eq. (6)), enfin de comparer celui-ci avec le spectre mesuré ; si le modèle adopté est correct, les 2 spectres doivent se superposer, aux erreurs expérimentales près.

2.5 GÉNÉRALISATION À DES PARTICULES DE GÉOMÉTRIE VARIABLE. - Enfin, si la suspension contient des particules de caractéristiques géométri- ques différentes, cela se manifeste au niveau du réseau par des images de formes et d'orientations différentes. Dans la pratique, les suspensions colloïdales ou biologiques étudiées sont assez homogènes en forme et dimension, et c'est donc principalement l'orientation différente des particules qui entraîne la poly-dispersité des images, celles-ci apparaissant comme des anneaux plus ou moins larges, circulaires ou elliptiques, et orientés de manière aléatoire. Le spectre de puissance est obtenu alors par une double intégration sur la vitesse et sur l'ensemble des images. On aboutira à une équation analogue à l'équation (6) à condition que les intégrations se fassent séparément, c'est-à-dire si la vitesse et la forme des images sont parfaitement indépendantes : 


$$
\begin{aligned}
P(F)=h\left(\frac{N \Lambda}{\pi}\right)^{2} \overline{\varphi^{2}} \int \frac{n_{\mathrm{s}}(v)}{v} \times & \left(\frac{\sin \pi\left(F-\frac{v}{\Lambda}\right) \frac{N \Lambda}{v}}{\pi\left(F-\frac{v}{\Lambda}\right) \frac{N \Lambda}{v}}\right)^{2} \mathrm{~d} v
\end{aligned}
$$$$
(\text { pour } p=1, F>0, m=2 \text { et } N \gg 1 \text { ) }
$$

où $\overline{\varphi^{2}}$ est la moyenne statistique du carré du flux incident dû à une particule, et $g_{v}^{2}(F)$ une fonction de la fréquence, résultant de l'intégration de la fonction $f^{2}(F)$ sur l'ensemble des images, de valeur $\leqslant 1$, et de variation lente par rapport au facteur $(\sin x / x)^{2}$.

\section{Dispositif expérimental.}

Schématiquement, l'appareil peut être dissocié en trois parties : le système optique, la cuve de mesure avec génération du champ électrique, et le système d'acquisition et de traitement du signal obtenu.

L'ensemble mécanique et optique est disposé sur une table anti-vibratoire réalisée au laboratoire à partir d'une table en granit Microcontrôle (GL 128). Celle-ci est placée sur une série de chambres à air communicantes.

3.1 SYSTÈME OPTIQUE. - La figure 7 donne une représentation schématique $\mathrm{du}$ dispositif optique utilisé.

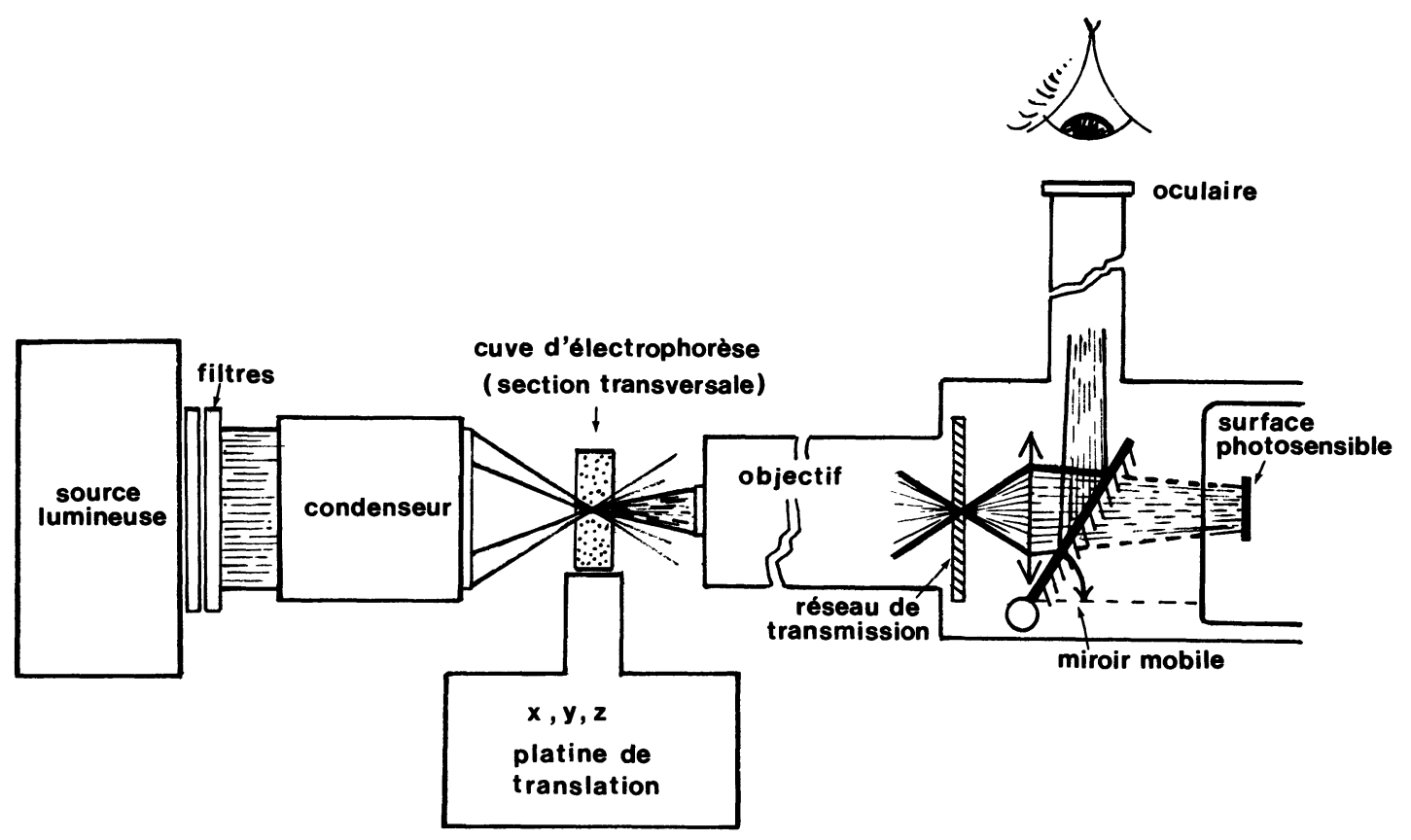

Fig. 7. - Schéma du dispositif optique.

[General layout of the optical part.]

3.1.1 Le couple objectif-condenseur travaillant en fond noir (équivalent d'un microscope à axe optique horizontal), a été réalisé selon nos spécifications par la Société CERCO.

Le faisceau lumineux sortant du condenseur peut être concentré dans la zone de mesure appropriée de la cuve. L'objectif a une ouverture numérique variable (maximum 0,3 ) inférieure à celle du condenseur (réglable de 0,31 à 0,48 ), si bien qu'il ne reçoit pas la lumière directe, mais seulement la lumière diffusée par les particules en suspension (Fig. 7). A l'oculaire, celles-ci apparaîtront donc brillantes sur fond noir.

La grande distance frontale de lobjectif $(\approx 10 \mathrm{~mm})$ rend possible l'observation dans toute la profondeur de la cellule. Son grandissement est faible, ce qui assure un champ optique transversal suffisant. L'objectif étant fixe, la mise au point se fait par le déplacement micrométrique de la cuve.

Ce système optique assure une très faible profondeur de champ $(\approx 15 \mu \mathrm{m})$, avec un faible grandissement $(G \approx 15)$. La distorsion de celui-ci est inférieure à $1 \%$ sur tout le champ image, ce qui est indispensable si l'on veut une résolution théorique du même ordre de grandeur.

L'ensemble précédent est maintenu sur un support métallique (banc optique) qui comporte également :

- un porte-réseau amovible pouvant tourner autour de l'axe optique, afin de choisir l'orientation relative des traits du réseau et de la direction de la vitesse ;

- un support pour le photodétecteur, muni d'une lentille de champ permettant d'éclairer la surface sensible du photodétecteur, de $10 \mathrm{~mm}$ de diamètre ; 
- une visée sur la surface sensible, afin d'optimaliser la focalisation;

- une visée réflexe oculaire permettant de faire les différents réglages optiques, et de contrôler régulièrement la bonne qualité et les mouvements de l'image formée sur le réseau. Un miroir mobile permet de commuter le faisceau lumineux soit vers la photocathode, soit vers l'oculaire.

3.1.2 Le réseau de transmission est fait par dépôt métallique sur une plaque de verre (Société D.M.E.). Celui utilisé en routine comporte $N=100$ fentes transparentes (verre). Ses caractéristiques géométriques ont été choisies en vue d'étudier des particules dont les dimensions sont de l'ordre du micromètre (entre 0,5 et 20 environ) : $h=7 \mathrm{~mm}$, $\Lambda=120 \mu \mathrm{m}, w=60 \mu \mathrm{m}(m=2)$. Cette valeur de $m$ permet d'éliminer tous les harmoniques d'ordre pair dans le spectre (Eq. (4)).

Sur le réseau, de surface $(12 \times 7) \mathrm{mm}^{2}$, se forme donc l'image de particules contenues dans un volume de dimensions $(0,8 \times 0,5 \times d) \mathrm{mm}^{3}(G \approx 15)$, où $\mathrm{d}$ est la profondeur de champ. Ce paramètre dépend de l'ouverture de l'objectif ; on peut le choisir aussi faible que $15 \mu \mathrm{m}$ environ.

3.1.3 La source lumineuse est une lampe quartzhalogène $50 \mathrm{~W}-12 \mathrm{~V}$ (luminaire Oriel 6324). La tension est fournie par une alimentation stabilisée modifiée (Adtech modèle APS 15-6).

La lumière blanche émise est filtrée par un filtre anticalorique (Oriel 5740) et un filtre interférentiel (Oriel G 572-5461) transmettant à $546,1 \mathrm{~nm}$, puis elle atteint un disque diffusant placé à l'entrée du condenseur.
3.1.4 Toute la lumière transmise à travers le réseau est reçue par une lentille de champ, puis atteint la surface sensible d'un photodétecteur, qui est une photodiode (type UDT $500 \mathrm{D}$ ), alimentée en $\pm 15 \mathrm{~V}$. L'emploi d'un photomultiplicateur, justifié quand on travaille avec de très faibles intensités lumineuses, s'est avéré inutile dans nos expériences.

3.2 CuVE D'ÉleCtRophorèse. - La partie critique de tout système d'électrophorèse est la cuve, dont les caractéristiques idéales sont les suivantes: volume utile minimum (afin d'être adapté aux échantillons étudiés, dont la quantité disponible est souvent très faible en biologie), absence d'artefacts au niveau des électrodes, possibilité d'appliquer un champ électrique fort sans convection ou échauffement excessifs, et facilité de nettoyage.

Nous avons adopté une cellule de section rectangulaire, à orientation latérale, qui convient bien à l'étude des éléments figurés du sang [37].

3.2.1 La cuve, réalisée par les établissements Hellma selon nos indications, est fabriquée en verre Pyrex, les faces latérales étant de qualité optique. Ses dimensions, dont le choix résulte d'un compromis [37], sont reportées sur la figure 8.

Elle est constituée d'une partie rectangulaire munie de deux tubulures latérales de section régulière, terminées par une coupe nette. Elle s'adapte (latéralement) entre deux blocs transparents en plexiglass où viennent $s$ 'emboîter de manière étanche les deux électrodes en $\mathrm{Ag} / \mathrm{AgCl}$ (disques plans fixés sur des cylindres en plexiglass). Le volume utile de liquide est d'environ $1 \mathrm{ml}$. L'ensemble est fixé sur un
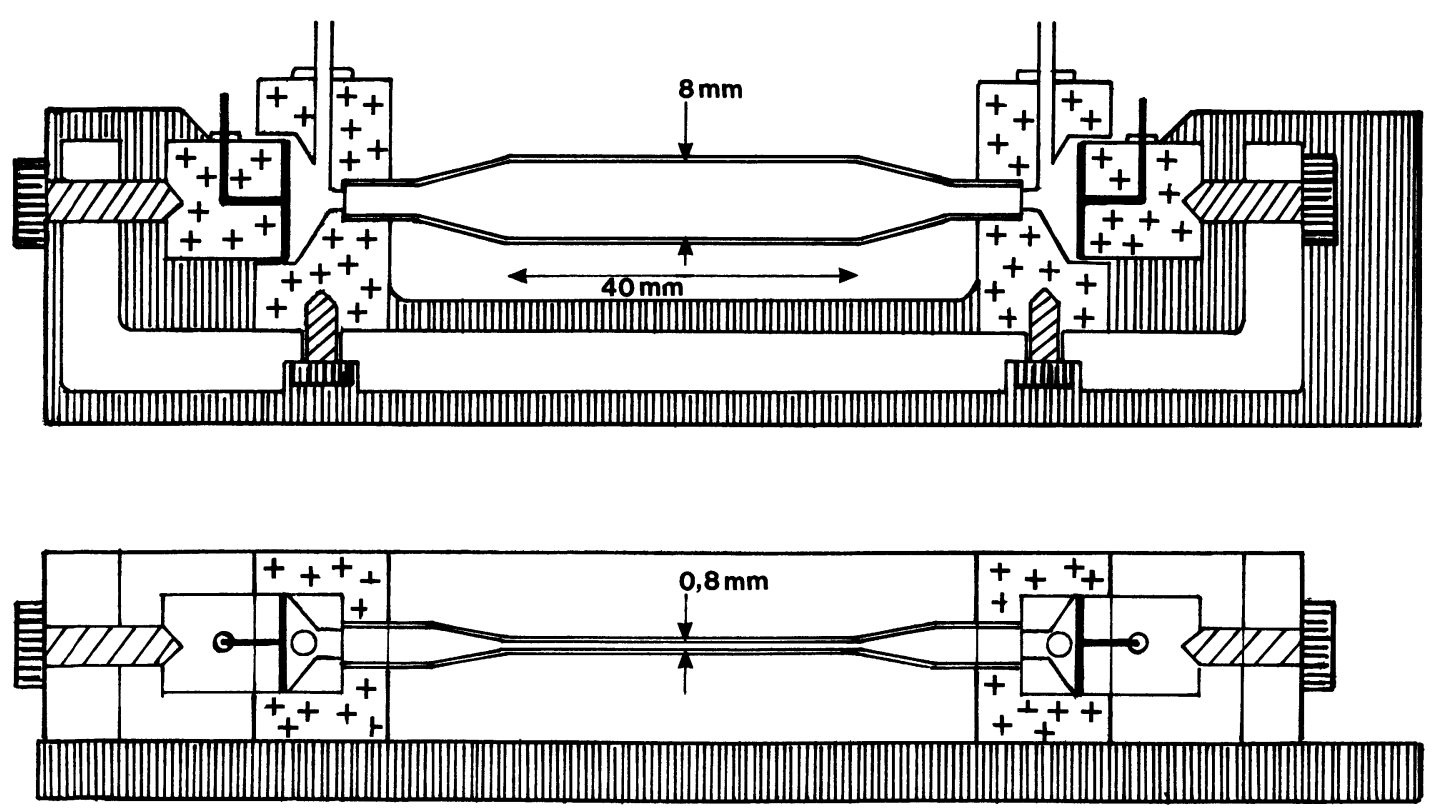

Fig. 8. - Cuve d'électrophorèse (dimensions en $\mathrm{mm}$ ).

[Electrophoretic cell (sizes in $\mathrm{mm}$ ).] 
support en Dural qui s'adapte sur un bloc de translation micrométrique (Microcontrôle).

Une fois installée sur le bloc Microcontrôle, la cuve est remplie in situ par l'intermédiaire d'un petit tube vertical (débouchant dans le compartiment d'électrode), la vidange se faisant de manière symétrique par l'autre compartiment (voir Fig. 8). Un système simple de robinets à plusieurs voies permet de faire s'écouler les suspensions par simple gravité. Pendant les mesures, les tubes d'entrée et de sortie de la cuve sont fermés, afin d'assurer l'étanchéité de l'ensemble.

Le bloc Microcontrôle permet la translation dans les 3 directions de l'espace. Le mouvement horizontal selon l'axe optique permet la mise au point sur un plan quelconque, à distance connue des parois de la cuve. Les deux autres mouvements, perpendiculaires à l'axe optique, sont utilisés pour sélectionner la région observée dans le plan de mise au point; de plus, le micromètre entraînant le déplacement horizontal perpendiculaire à l'axe optique est équipé d'un moteur à vitesse constante (Escap) de façon à pouvoir étalonner le système.

La cuve n'est pas thermostatée, les résultats étant ramenés à $25^{\circ} \mathrm{C}$ comme nous l'expliquons ultérieurement. Les gradients de température sont évités en réalisant une section transversale à peu près constante $\left(\sim 6,5\right.$ à $\left.7 \mathrm{~mm}^{2}\right)$ dans toute la cuve (partie rectangulaire et tubulures latérales), ce qui limite les courants de convection thermique.

3.2.2 Les électrodes sont de type réversible, en $\mathrm{Ag} / \mathrm{AgCl}$. Elles sont obtenues par chloruration d'une couche d'argent pur.

Dans la plupart de nos expériences d'électrophorèse, nous utilisons des solutions de faible conductivité, ce qui réduit la densité de courant à la surface des électrodes $(I / S=\sigma E)$, et donc limite tout phénomène résiduel de polarisation, ou de dégagement gazeux. De plus, la distance entre électrodes est suffisante pour éliminer tout risque de contamination de la zone d'observation de la cuve pendant la durée d'application du champ électrique $(\sim 20 \mathrm{~s})$.

Remarque : au voisinage des parois en verre de la cellule, en contact avec le milieu de suspension, il se crée une double couche électrique [40,5] dont les ions mobiles vont se mettre en mouvement sous l'action du champ électrique, entraînant par viscosité le liquide qui les entoure. Cet écoulement de liquide le long des parois de la cuve (phénomène d'électroosmose) est nécessairement équilibré par un flux de liquide en sens inverse à travers le milieu de la cellule (système fermé) : il en résulte un certain profil de vitesses, qui perturbe les mesures. Il est indispensable de déterminer avec précision les plans stationnaires de la cuve, où la vitesse d'entraînement du liquide $(u)$ est nulle, la vitesse mesurée dans ces plans correspondant alors à la vitesse électrophoréti- que absolue $(v)$ des particules. Pour la géométrie choisie, les plans stationnaires sont donnés par la théorie de Komagata [41].

Certains auteurs ont choisi de limiter le flux électroosmotique, soit en recouvrant les parois de la cuve avec un produit électriquement neutre au contact de l'eau [20, 42-44], soit en réduisant le champ électrique le long des parois par une géométrie appropriée [18] ; ces procédés ne sont pas sans inconvénient [37] et nous avons préféré tenir compte du phénomène d'électroosmose en le contrôlant, c'est-à-dire en déterminant le profil électroosmotique et les plans stationnaires correspondants.

3.2.3 Génération du champ électrique : comme la distance entre les électrodes est élevée $(\approx 10 \mathrm{~cm})$, il est indispensable de disposer d'un générateur de haute tension pour créer un champ électrique suffisant au sein de la cellule de mesure.

Les électrodes sont connectées à une source de courant programmable (amplificateur haute tension Kepco modèle BOP $1000 \mathrm{M})$, alimentée par un générateur d'impulsions rectangulaires de tension $(0,01$ à $9,99 \mathrm{~V})$, réalisé selon nos spécifications par le laboratoire I.N.S.E.R.M. SCR 3 de Nancy. L'amplificateur délivre ainsi des créneaux de courant imposé, de longueur, d'amplitude et de polarité variables. Ses caractéristiques limites sont : $-1000 \mathrm{~V}$ à $+1000 \mathrm{~V},-40 \mathrm{~mA}$ à $+40 \mathrm{~mA}$, durée 0,1 à $99,9 \mathrm{~s}$.

L'amplitude du champ électrique s'obtient directement à partir de la loi d'Ohm $(E=I / \sigma S)$. Calculée par cette méthode, sa valeur reste indépendante d'une éventuelle polarisation des électrodes. I est l'amplitude du courant électrique traversant la cellule ; elle est mesurée par un ampèremètre (multimètre digital Fluke $8000 \mathrm{~A}$ ) placé en série entre la cuve d'électrophorèse et la source de courant. $S$ est la section transversale de la cuve d'électrophorèse, déterminée optiquement à l'aide des micromètres du bloc Microcontrôle $\left(S=6,48 \mathrm{~mm}^{2}\right)$; la mesure de $S$ dans la zone même d'observation des particules permet de connaître le champ électrique exact dans cette zone. $\sigma$ est la conductivité de la suspension; elle est mesurée séparément à température connue (en général $25^{\circ} \mathrm{C}$ ) à l'aide d'une micro-cellule à aspiration [45], reliée à un conductimètre numérique de précision réalisé au laboratoire. Les milieux à mesurer sont maintenus dans un thermostat stabilisé à mieux de $0,1^{\circ} \mathrm{C}$.

\subsection{ACQUiSITION ET TRAITEMENT DU SIGNAL.}

3.3.1 Mise en forme du signal. - Le photodétecteur délivre un photocourant (Fig. 9) dont la modulation représente le signal intéressant. Il est filtré (pour supprimer la composante continue inutile, et éventuellement des hautes fréquences parasites $>1 \mathrm{kHz}$ ) et amplifié ( $\times 10$ à $\times 10 \mathrm{k})$, par un préamplificateur 


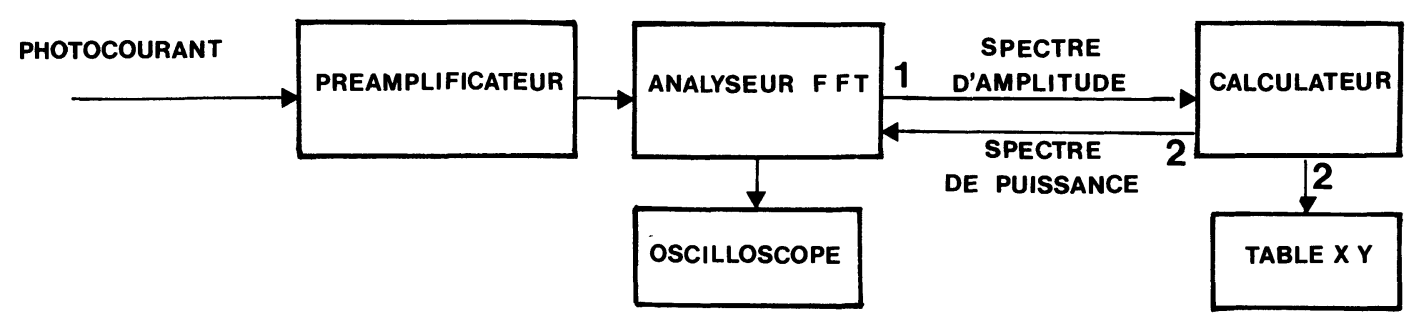

Fig. 9. - Schéma de l'ensemble d'acquisition et de traitement du signal.

[Block diagram of the signal processing set up.]

à faible bruit (Princeton Applied Research Corporation modèle 113). On obtient ainsi une tension alternative, proportionnelle à la partie alternative du flux lumineux, $\Phi(t)-\langle\Phi(t)\rangle$.

3.3.2 Analyse du signal. - Le signal issu du préamplificateur (Fig. 9) est adressé alors à un analyseur de spectre en temps réel, qui en fait la transformée de Fourier rapide (FFT). L'analyseur (Princeton Applied Research modèle 4513) présente une grande dynamique d'entrée $(70 \mathrm{~dB})$ et une grande résolution spectrale (1 024 ou 2048 points de fréquence). En général les mesures sont faites sur la gamme 0$50 \mathrm{~Hz}$, ce qui nécessite un temps d'acquisition du signal de $20 \mathrm{~s}$ (pour 1024 points d'analyse).

Afin de n'analyser que le signal utile, dû au champ électrique, il faut que la durée d'acquisition $T$ du signal soit légèrement inférieure au temps d'application du champ électrique : $T=1 / \Delta f \leqslant \Delta t$. D'autre part, il faut que la bande passante $\Delta f=1 / T$ de l'analyseur soit au plus égale à la largeur théorique des pics $F_{1} / N$. Il faut donc choisir le temps d'acquisition $T$ du signal dans le domaine $N / F_{1} \leqslant T \leqslant \Delta t$.

3.3.3 Calcul du spectre de puissance. - Le spectre d'amplitude fourni par l'analyseur FFT (Fig. 9) est ensuite adressé à un calculateur Hewlett-Packard (type $9825 \mathrm{~A}$ ) par l'intermédiaire d'un interface réalisé au laboratoire. Il y est alors traité (converti en spectre de puissance) et stocké, puis adressé de nouveau soit vers l'analyseur de spectre pour visualisation sur l'oscilloscope associé, soit vers une table traçante Hewlett-Packard (type $7225 \mathrm{~A}$ ).

La vitesse d'une particule est reliée à la fréquence par la relation $v=F \Lambda / G$, ou au canal correspondant $X$ sur l'analyseur par:

$$
v=\frac{f_{\mathrm{R}}}{n} X \frac{\Lambda}{G}
$$

où $f_{\mathrm{R}}$ est la gamme des fréquences analysées, et $n$ est le nombre de points d'analyse (1 024 ou 2048 ); dans nos expériences, on a en général $f_{\mathrm{R}} / n=$ $0,05 \mathrm{~Hz}$.

\section{Protocole expérimental.}

Nous décrirons ici complètement le déroulement d'une mesure de mobilité électrophorétique sur une suspension d'hématies. Ceci n'a évidemment que valeur d'exemple. Lorsque les mesures sont répétitives, à l'occasion d'une étude particulière, il va sans dire que le protocole se simplifie et que le temps de manipulation est d'autant raccourci. De plus, ce protocole serait également beaucoup plus court dans d'autres applications du VRT, telles que la mesure de la vitesse de particules dans des écoulements liquides ou gazeux.

4.1 Préparation et CARACTÉRISATION DES SUSPENSIONS. - Une suspension fraîche d'hématies est préparée par centrifugation de tubes de sang prélevé sur EDTA ou sur héparine, puis (éventuellement) lavage des hématies avec le milieu de mesure, et resuspension dans ce milieu.

Le choix de la concentration finale des suspensions mesurées doit obéir à certains critères : des concentrations trop élevées sont à éviter parce que le fond continu de diffusion augmente, ce qui réduit alors la valeur relative de la modulation ; pour des concentrations trop faibles au contraire, la modulation devient du même ordre de grandeur que le bruit, et le signal ne peut plus être considéré comme stationnaire.

La plage de concentration utile pour les hématies est de $0,1 \times 10^{-3}$ à $5 \times 10^{-3}$ en volume. Il est inutile d'obtenir une concentration donnée avec précision, car pour de si grandes dilutions, la mobilité est indépendante de la concentration. La concentration habituellement utilisée de $0,1 \%$ en volume correspond à une concentration numérique voisine de $10^{4}$ cellules $/ \mathrm{mm}^{3}$. Au niveau du réseau on aura alors un nombre moyen de particules-images par unité de surface $N_{\mathrm{s}}=10^{4} d_{(\mathrm{mm})} / G^{2}$, soit par exemple $N_{\mathrm{s}}=$ 102 cellules $/ \mathrm{cm}^{2}$ avec $G=14$ et $d=20 \mu \mathrm{m}$, d'où un nombre moyen de particules-images sur le réseau $=102 \times 0,84 \approx 86$. Cet exemple numérique indique que pendant une mesure, plus d'une centaine (200 ou 300) de particules-images défileront sur le réseau, ce qui est raisonnable pour assurer une signification statistique aux résultats de mobilité.

En règle générale, nous avons limité la conductivité des suspensions à $1 \mathrm{mS} . \mathrm{cm}^{-1}$ environ, afin d'éviter des élévations de température à l'origine de courants de convection aléatoires; c'est pourquoi 
nous travaillons à faible force ionique $\left(10^{-2} \mathrm{M}\right.$ maximum). Des valeurs plus élevées peuvent être utilisées [37], mais nous n'avons pas fait d'étude systématique dans ce sens, notre objectif étant d'assurer les conditions expérimentales optimales en vue d'obtenir la meilleure résolution spectrale possible.

La température de la suspension n'est pas maintenue constante. Les dispositifs de contrôle souvent utilisés ont une efficacité illusoire à notre avis, car (tout au moins lorsque des mesures rapides sont nécessaires) le temps caractéristique de transfert thermique à travers la cuve et la suspension est du même ordre de grandeur, ou plus grand, que la durée de la mesure : ainsi la température de la suspension n'est pas identique à celle du liquide thermostatique, ce qui est susceptible d'entraîner des erreurs notables lorsque l'effet Joule est important. Toutefois, lorsque les mesures d'électrophorèse se font à courant imposé, les variations de température au cours de la mesure (dues à l'effet Joule par exemple), sont sans influence sur les résultats : en effet, quand on travaille à courant constant $I$, toute variation de température entraîne une variation de la conductivité donc du champ électrique $E$ ( $E=$ $I / \sigma S \sim 1 / \sigma)$; par ailleurs, la mobilité $\mu$ varie avec la température comme la conductivité $(\mu \sim \sigma)$ [37] : la vitesse mesurée $v=\mu E$ reste donc constante. C'est pourquoi on peut faire les mesures à la température ambiante, sans se soucier de sa valeur exacte. Pour obtenir la mobilité ramenée à une température de référence $\left(25^{\circ} \mathrm{C}\right)$, il suffira alors de calculer le champ électrique $E$ à cette même température à partir de la conductivité correspondante de la suspension, $\sigma_{25}$. D'où : $\mu_{25}=\left(\sigma_{25} S / I\right) v$.

4.2 DÉROULEMENT D'UNE MESURE. - Le dispositif optique est mis au point : éclairage en fond noir avec la plus grande ouverture possible de l'objectif, réglage du condenseur par focalisation du faisceau incident sur le plan de mise au point (par exemple sur la face externe arrière de la cuve, côté condenseur), réglage de la bague de correction d'aberration sphérique, visée sur la photocathode pour apprécier l'alignement optique, et enfin vérification de l'orientation de la trame, dont les fentes doivent être perpendiculaires à la vitesse, c'est-à-dire verticales.

La cellule d'électrophorèse, obturée avec les 2 électrodes, est rincée plusieurs fois avec le solvant, puis remplie avec la suspension étudiée, en évitant d'y introduire des bulles d'air, qui entraineraient des mouvements de dérive. Une fois installée correctement sur son support, la cuve sera remplie in situ pour les expériences suivantes, sans modification du réglage. La suspension observée à l'oculaire doit apparaître immobile.

La position le long de l'axe optique des deux faces internes verticales de la cuve est déterminée par mise au point sur ces faces et lecture du micromètre de translation. L'épaisseur apparente de la cuve étant ainsi connue $(610 \mu \mathrm{m})$, la position des deux plans stationnaires de Komagata et du plan médian se calcule aisément [37].

Ayant choisi le plan de mise au point dans la suspension, le gain de la photodiode, les gains d'entrée du préamplificateur et de l'analyseur de spectre sont ajustés de manière à avoir un signal suffisant au niveau de l'analyseur, tout en évitant sa saturation.

On choisit sur le module de commande l'intensité $I$ et la durée $\Delta t$ du courant électrique. Un contrôle à l'oculaire permet de vérifier que les particules se déplacent bien sous l'action du champ électrique, sans mouvement erratique, et que toutes les fentes du réseau sont traversées pendant la durée $\Delta t$. On déclenche alors le courant électrique puis l'analyse spectrale ; une seule acquisition est nécessaire $(20 \mathrm{~s}$ pour 1024 points d'analyse, dans la gamme 0$50 \mathrm{~Hz}$ ). Le contenu de. l'analyseur (spectre d'amplitude) est ensuite adrèssé vers le microordinateur, qui calcule le spectre de puissance correspondant, et le visualise sur l'oscilloscope ou sur la table traçante ; il calcule aussi la fréquence moyenne du spectre dans une zone de fréquences choisie.

La mobilité électrophorétique moyenne d'une suspension est calculée à partir des spectres de puissance obtenus dans les deux plans stationnaires : le calculateur détermine les canaux moyens correspondants (soit $\mathrm{Y}$ et $\mathrm{Z}$ ), puis la mobilité moyenne à $25^{\circ} \mathrm{C}$ par la formule :

$$
\overline{\mu_{25}}=\frac{\sigma_{25} S}{I} \frac{\Lambda}{G} \frac{f_{\mathrm{R}}}{n} \frac{Y+Z}{2} .
$$

La distribution des mobilités électrophorétiques est obtenue en général avec une meilleure résolution dans le plan médian de la cuve, là où le gradient de vitesse électroosmotique est nul. Le calculateur décale le spectre de puissance médian (canal moyen $X)$ dans le sens négatif, par translation de tous les canaux $X_{i}$ de la quantité $X-(Y+Z) / 2$; le spectre résultant, centré sur le canal $(Y+Z) / 2$, représente alors le spectre des vitesses électrophorétiques $v_{i}$, ou des mobilités électrophorétiques $\mu_{i, 25}$ :

$$
\mu_{i, 25}=\frac{\sigma_{25} S}{I} \frac{\Lambda}{G} \frac{f_{\mathrm{R}}}{n}\left[X_{i}-X+\frac{Y+Z}{2}\right] .
$$

On a ainsi une image exacte de la distribution des mobilités, en s'affranchissant des effets électroosmotiques.

\section{Résultats.}

Afin de tester le dispositif expérimental, il est nécessaire d'utiliser une suspension de particules pour laquelle la dispersion des mobilités soit la plus 
faible possible. Le globule rouge humain est un bon modèle de particule, car en plus de la constance de sa mobilité (dans un milieu déterminé), il est très facile à obtenir et à mesurer; nous l'avons donc choisi comme particule standard pour étalonner notre vélocimètre.

Différents types d'études ont été réalisés sur le montage vélocimétrique à titre de contrôle, avec ou sans champ électrique.

5.1 Études À VITESSE CONSTANTE. — La figure 10 montre le signal modulé dans le temps (à la sortie du préamplificateur), obtenu lorsque la cuve (contenant des globules rouges en suspension diluée) est déplacée à vitesse constante selon une direction horizontale perpendiculaire à l'axe optique. Le spectre de puissance résultant est donné figure 11. A partir de la valeur de la fréquence fondamentale $F_{1}$, et de la vitesse de translation micrométrique $v$, on détermine le facteur d'étalonnage du système, $F_{1} / v$ (en $\left.\mathrm{cm}^{-1}\right)$ :

$F_{1}=14,75 \mathrm{~Hz}$

$$
v=125 \mu \mathrm{m} / \mathrm{s} \Rightarrow F_{1} / v=1180 \mathrm{~cm}^{-1} .
$$

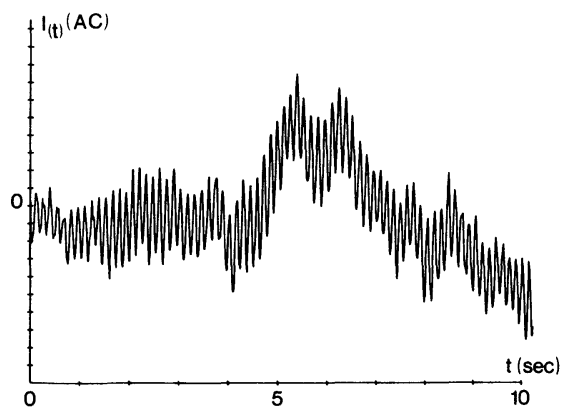

Fig. 10. - Composante alternative du signal modulé, la cuve étant translatée à vitesse constante $v$.

[Time-modulated signal (ac), the cuvette being moved at constant speed $v$.]

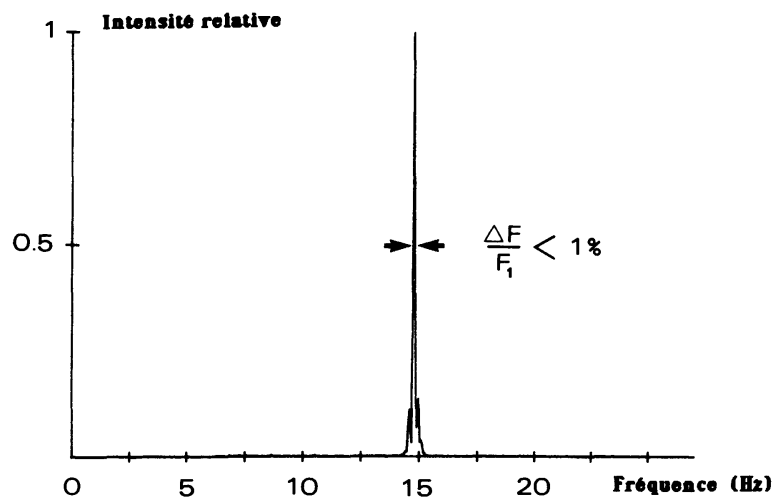

Fig. 11. - Spectre de vitesses des hématies, la cuve étant translatée à vitesse constante $v: F_{1}=G v / \Lambda$.

[Spectrum of the erythrocytes velocity, the cuvette being moved at constant speed $v: F_{1}=G v / \Lambda$.]
Tableau I. - Contenu des canaux de l'analyseur pour le spectre de la figure 11.

[Content of analyser's canals for the figure $11 \mathrm{spec}-$ trum.]

\begin{tabular}{|c|r|}
\hline Canaux & Intensité \\
\cline { 2 - 3 }$F_{1}=14,75 \mathrm{~Hz} \rightarrow \quad 289$ & 25 \\
290 & 138 \\
291 & 224 \\
292 & 107 \\
293 & 67 \\
294 & 896 \\
295 & 2047 \\
296 & 714 \\
297 & 138 \\
298 & 147 \\
299 & 280 \\
300 & 67 \\
301 & 73 \\
\hline
\end{tabular}

L'analyse se fait sur 1024 canaux, dans la gamme de fréquences $0-51,2 \mathrm{~Hz}(0,05 \mathrm{~Hz}$ par canal).

Le tableau I indique le contenu des canaux de l'analyseur (normé à 2047) pour le spectre de la figure 11 , de part et d'autre de la fréquence fondamentale $F_{1}$.

On peut voir sur le spectre de la figure 11, ou sur le tableau I, que la résolution obtenue est de l'ordre de $1 \%$, donc très proche de la résolution théorique $1 / N$ attendue avec un réseau de 100 fentes (cf. Fig. $\left.4: \Delta F / F_{1} \approx 0,9 / N\right)$.

On remarque l'existence de lobes latéraux, centrés sur les canaux 291 et 299 (fréquences 14,55 et $14,95 \mathrm{~Hz}$ ), donc à $0,2 \mathrm{~Hz}$ de part et d'autre du pic central $F_{1}=14,75 \mathrm{~Hz}$. Ces lobes confirment la formule théorique (4): ils correspondent en effet aux maxima de la fonction $(\sin x / x)^{2}$, où $x=$ $\pi(F-V / \Lambda) N \Lambda / V$, les premiers maxima ayant lieu pour $x= \pm 1,43 \pi$, c'est-à-dire pour $F-F_{1}=$ $\pm 1,43 F_{1} / N= \pm 0,21 \mathrm{~Hz}$.

Remarquons que l'amplitude de ces lobes latéraux est toujours faible comparée à celle du pic fondamental : la théorie prévoit qu'elle est de l'ordre de $5 \%$ de l'amplitude du pic $F_{1}$. Lors de l'étude pratique de particules en suspension, ces lobes ne risquent d'apparaître que si la distribution des vitesses est très étroite, ce qui n'arrive qu'exceptionnellement; et même dans ce cas, une correction simple sur le spectre permet de les éliminer (déconvolution).

D'autres réseaux ont été testés afin de vérifier la relation $F_{1}=G v / \Lambda$, en conservant la même vitesse de translation de la cuve $\left(125 \mu \mathrm{m} . \mathrm{s}^{-1}\right)$. Les résultats sont reportés sur la figure 12 . 


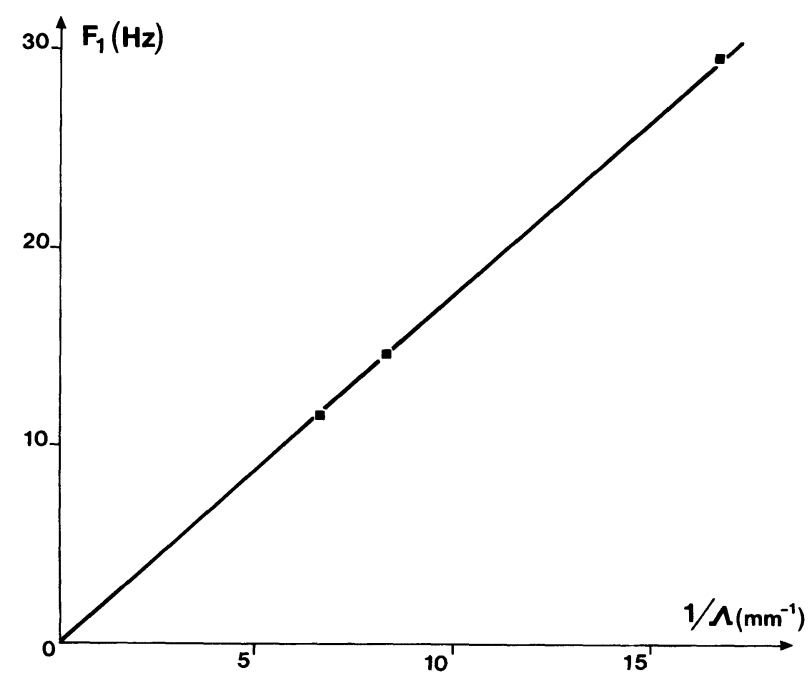

Fig. 12. - Variation de la fréquence fondamentale $F_{1}$ avec l'inverse de la période spatiale $\Lambda$.

[Plot of the fundamental frequency $F_{1}$ versus the spatial period $\Lambda$ reciprocal.]

La courbe $F_{1}=f(1 / \Lambda)$ est bien une droite passant par l'origine. A partir de la pente de cette droite, on peut calculer de manière précise le grandissement optique $G=F_{1} \Lambda / v$; on le trouve égal à 14,16 à $1 \%$ près, ce qui confirme la valeur déduite de l'observation à l'oculaire du réseau (de dimensions connues), en s'aidant des translations micrométriques $x$ ou $y$.

5.2 ÉtUde DE PROFIL DE VITESSE. - Nous avons déterminé les profils de vitesse dans la cuve rectangu- laire (épaisseur $2 h=0,8 \mathrm{~mm}$ ), lors d'un écoulement laminaire à débit constant dans la direction $O x$ (horizontale perpendiculaire à l'axe optique). Les globules rouges sont utilisés ici comme marqueurs pour visualiser le liquide (sérum physiologique), qui s'écoule par simple gravitation.

En régime stationnaire, la théorie prévoit dans la région centrale de la cuve $(x=y=0)$ une variation parabolique de la vitesse avec la profondeur $z$ $(-h \leqslant z \leqslant+h), \quad$ de la forme $v(z)=$ $v_{\max }\left(1-z^{2} / h^{2}\right), v_{\max }$ étant la vitesse maximum de l'écoulement, obtenue au centre $z=0$ de la cuve.

Pour une expérience donnée, nous explorons toute l'épaisseur de la cuve entre les 2 parois verticales, afin d'obtenir une trentaine de spectres de puissance, tous les $20 \mu \mathrm{m}$ environ. En représentant la fréquence moyenne de ces spectres en fonction du carré de la distance $z$, on obtient 2 séries de points expérimentaux, pour $-h<z \leqslant 0$ et $0 \leqslant z<+h$, qui s'alignent correctement sur une droite coupant l'axe des abscisses au point $|z|=h$ (Fig. 13).

Ces résultats confirment que le profil de vitesse est bien symétrique, parabolique, et que la vitesse s'annule au niveau des parois. La technique employée a permis d'obtenir le profil d'écoulement avec précision, y compris à proximité immédiate des parois, ce qui est une bonne vérification du caractère très local des mesures.

Par ailleurs, nous constatons que les spectres sont de plus en plus larges au fur et à mesure que l'on s'approche des parois, ce qui est dû au gradient de vitesse qui augmente avec $z$, d'où une distribution de vitesses plus large dans la tranche explorée d'épaisseur $d$ (profondeur de champ); la variation de vitesse correspondante est en effet: $\Delta v_{d}=$ $2\left(v_{\max } / h^{2}\right) d \times z$, proportionnelle à $z$.

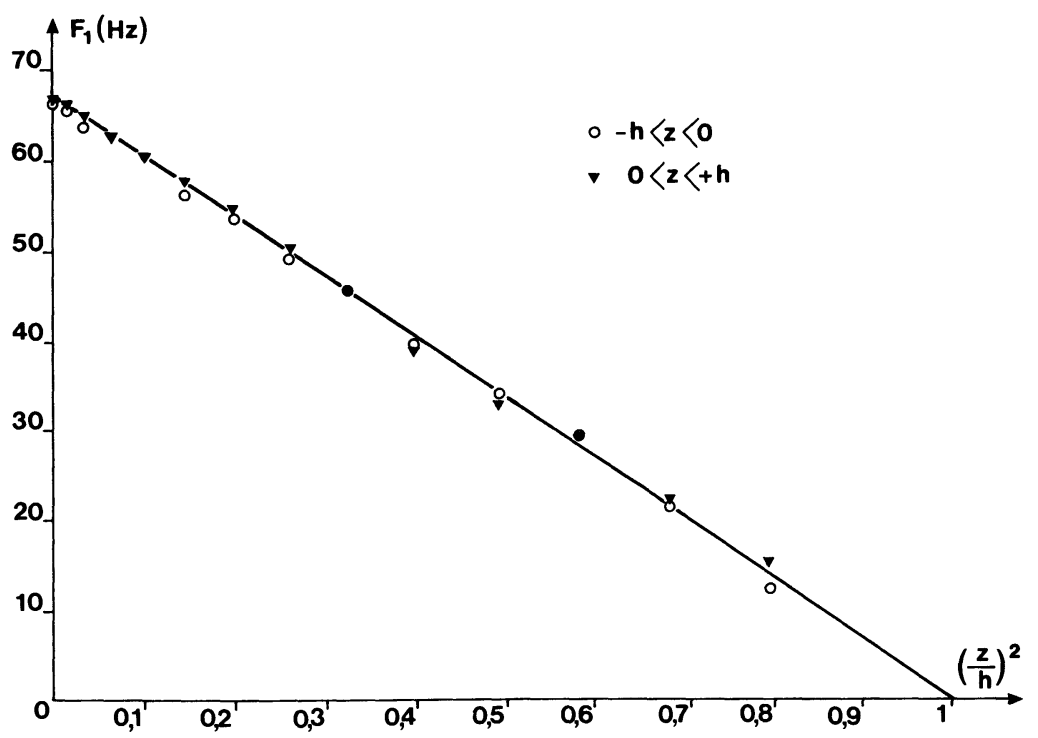

Fig. 13. - Variation de la fréquence fondamentale $F_{1}$ avec le carré de la profondeur $z$ dans la cuve (écoulement à débit constant).

[Variation of the fundamental frequency $F_{1}$ with the square of the depth $z$ in the cell (constant intensity flow).] 
Nous pouvons avoir une estimation de la profondeur de champ en mesurant la largeur à mi-hauteur de quelques spectres, en fonction de la distance $z$. L'élargissement étant dû en partie à la profondeur de champ $d$, en partie au nombre fini $N$ de fentes traversées $(\Delta v / v \approx 1 / 100)$, nous supposons pour simplifier que les largeurs correspondantes s'ajoutent :

$$
\Delta v_{\text {spectrc }}=\Delta v_{d}+\Delta v_{\text {fentes }}
$$

ou

$$
\left(\Delta v_{d} / v_{\max }\right)=\left(\Delta v_{\mathrm{sp}} / v_{\max }\right)-v / 100 v_{\max } .
$$

Le tableau II donne les résultats obtenus pour quatre valeurs de $z$. En portant sur un graphique $\Delta v_{d} / v_{\max }$ en fonction de $z_{\text {apparent }}$ (Fig. 14), on obtient effectivement une droite, qui passe par l'origine. La profondeur de champ peut être calculée à partir de la pente de cette droite $\left(\Delta v_{d} / v_{\max }\right) / z=2 d / h^{2}$. D'où

$$
d_{\text {apparent }}=(0,033 / 100) \times(610)^{2} / 8=15,35 \mu \mathrm{m} .
$$

Compte tenu de l'indice de réfraction du liquide, la profondeur de champ réelle est donc de l'ordre de $20 \mu \mathrm{m}$.

Tableau II. - Estimation de l'élargissement spectral dû à la profondeur de champ.

[Estimation of the spectral broadening caused by the depth of field.]

\begin{tabular}{|c|c|c|c|}
\hline $\begin{array}{c}Z(\mu \mathrm{m}) \\
\text { apparent }\end{array}$ & $\begin{array}{l}v / v_{\max } \\
=1-z^{2} / h^{2}\end{array}$ & $\frac{\Delta v_{\mathrm{sp}}}{v_{\max }}$ & $\frac{\Delta v_{d}}{v_{\max }}$ \\
\hline 20 & 0,996 & 0,0156 & 0,0056 \\
40 & 0,983 & 0,0249 & 0,0151 \\
60 & 0,961 & 0,0311 & 0,0215 \\
100 & 0,893 & 0,0404 & 0,0315 \\
\hline
\end{tabular}

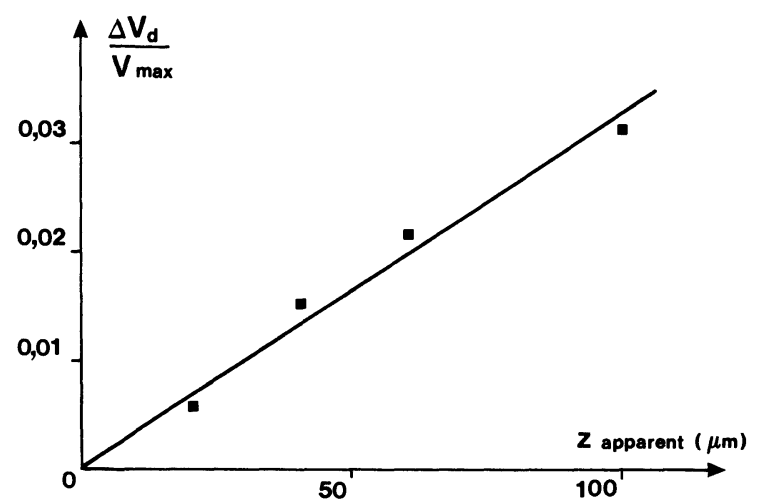

Fig. 14. - Variation de la largeur spectrale $\Delta v_{d} / v_{\max }$ en fonction de la profondeur $z$ dans la cuve.

[Variation of the spectral width $\Delta v_{d} / v_{\max }$ as a function of the depth $z$ in the cell.]
Remarque : les globules rouges ayant un diamètre de l'ordre de $8 \mu \mathrm{m}$, on observe donc qu'une défocalisation de 1 à 1,5 diamètre fait diminuer de moitié l'intensité du spectre, ce qui indique que la contribution des particules hors mise au point est rapidement négligeable.

5.3 Études AVEC CHAMP ÉlectriQue. - Avant de commencer nos études électrophorétiques sur les hématies, nous avons voulu vérifier que la mobilité était bien indépendante du champ électrique appliqué. La figure 15 montre les résultats obtenus sur 3 suspensions différentes d'hématies (en tampon phosphate $10^{-2} \mathrm{M}$ ) lorsque l'on fait varier l'intensité du courant électrique. Dans chaque cas, la fréquence fondamentale $F_{1}$ mesurée est bien proportionnelle à l'intensité du courant $I$, ce qui confirme la constance de la mobilité, qui est à une constante multiplicative près la pente de chaque droite $\left[F_{1}=(G \mu / \Lambda \sigma S) I\right]$.

Ensuite nous avons procédé à un étalonnage de la cellule d'électrophorèse, qui consiste à vérifier la forme parabolique du profil électroosmotique et la position des plans de Komagata [37].

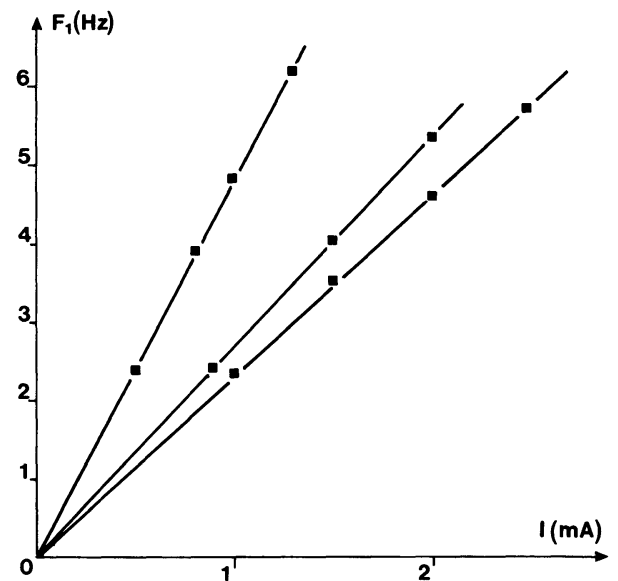

Fig. 15. - Variation de la fréquence fondamentale $F_{1}$ avec l'intensité $I$ du courant électrique, pour 3 suspensions différentes d'hématies.

[Plot of the fundamental frequency $F_{1}$, versus the electric current intensity $I$, for 3 different erythrocytes suspensions.]

Sur une même suspension de globules rouges, nous avons déterminé à courant constant une quinzaine de spectres électrophorétiques dans toute l'épaisseur $2 h$ de la cuve, tous les $40 \mu \mathrm{m}$ à partir de la face interne arrière (plus le plan $z=0$ ). En représentant la fréquence moyenne $F_{1}$ de ces spectres en fonction de la distance $z$ (correspondant à $\left.v_{\mathrm{obs}}=u(z)+v\right)$, on obtient des points expérimentaux qui se situent effectivement sur une parabole, légèrement décentrée (Fig. 16). La courbe théorique parabolique est déterminée à partir dès 15 points expérimentaux, par la méthode des moindres carrés. 


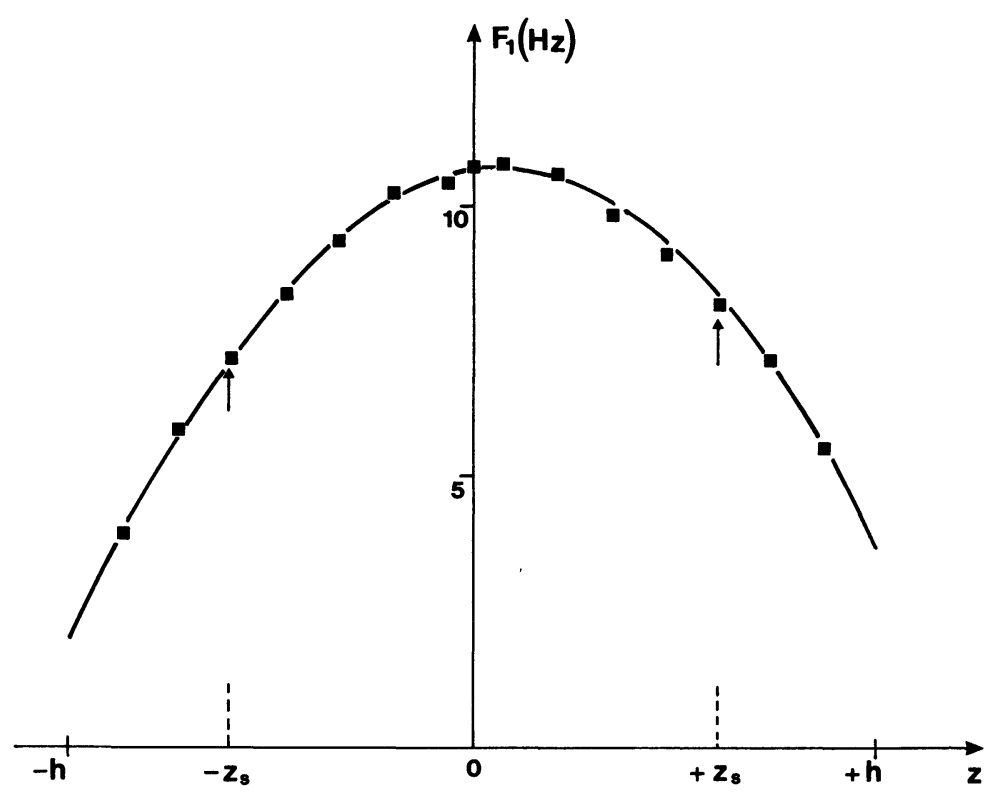

Fig. 16. - Variation de la fréquence moyenne $F_{1}$ en fonction de la profondeur $z$ dans la cuve : (-) courbe théorique (parabole),(ם) points expérimentaux.

[Variation of the mean frequency $F_{1}$, as a function of the depth $z$ in the cell : ( - ) theoretical curve (parabole), ( $\square$ ) experimental points.]

L'asymétrie du profil parabolique, observée par de nombreux auteurs [37], s'explique par une légère dissymétrie des densités de charge des parois. Les plans de Komagata ne sont plus alors «stationnaires ", puisque la vitesse d'entraînement $(u)$ du liquide n'y est plus nulle, mais proportionnelle à la différence des mobilités électroosmotiques des 2 parois. Dans les 2 plans de Komagata, les vitesses d'entraînement $u(z)$ sont de même valeur absolue et de signes contraires, si bien que par moyenne des vitesses observées $v_{\text {obs }}$ dans ces plans, on obtient la vitesse électrophorétique absolue $v$ des particules.

Il ne suffit pas d'avoir vérifié la variation parabolique de la vitesse pour affirmer que la mobilité moyenne des particules d'un échantillon donné s'obtient par moyenne des mesures réalisées dans les plans de Komagata. Pour vérifier expérimentalement la position de ces plans, on pourrait prendre une suspension de particules de mobilité connue et constante, et vérifier la valeur de cette mobilité dans les plans de Komagata. Une méthode plus générale [37] consiste à tracer la courbe expérimentale $F_{1}=f(z)$, en étudiant une suspension quelconque de particules, de mobilité inconnue, et à calculer la surface comprise entre la courbe et l'axe des $z$. En effet, la cellule étant fermée, que la fonction $v_{\text {obs }}(z)$ soit parabolique ou non, la condition de flux nul implique :

$$
\int_{-h}^{+h} v_{\text {obs }}(z) \mathrm{d} z=\int_{-h}^{+h} u(z) \mathrm{d} z+\int_{-h}^{+h} v \mathrm{~d} z=2 h v
$$

ou $\quad \int_{-h}^{+h} F_{1}(z) \mathrm{d} z=2 h F_{\mathrm{s}}$.

Ainsi, par planimétrie de la courbe de la figure 16, on obtient :

$$
F_{\mathrm{s}}=\int F_{1}(z) \mathrm{d} z / 2 h=7,90 \mathrm{~Hz} .
$$

D'autre part, dans les plans de Komagata indiqués sur la figure 16 par des flèches $(z= \pm 0,607 \mathrm{~h})$, on mesure : $F_{1}\left(+z_{\mathrm{s}}\right)=8,25 \mathrm{~Hz}$ et $F_{1}\left(-z_{\mathrm{s}}\right)=7,20 \mathrm{~Hz}$, d'où par moyenne $F_{\mathrm{s}}=7,73 \mathrm{~Hz}$. Les deux valeurs de $F_{\mathrm{s}}$ ainsi trouvées concordent à $2 \%$ près, ce qui constitue une bonne vérification des plans théoriques de Komagata.

Par cette méthode, nous avons donc étalonné la cuve d'électrophorèse, en contrôlant la validité de la formule de Komagata. Il est donc bien justifié de faire les mesures de mobilité moyenne dans ces plans. Remarquons qu'il est indispensable de faire les mesures dans les deux plans, car la parabole des vitesses est en général non symétrique par rapport à $z=0$.

5.4 Mobilité ÉLECTROPHORÉTIQUE DES GLOBULES ROUGES HUMAINS. - La charge négative nette des érythrocytes de la plupart des mammifères est due principalement aux groupements carboxyliques de l'acide sialique ; chez l'homme, il s'agit de l'acide $\mathrm{N}$-acétyl-neuraminique, qui est situé entièrement sur la surface extérieure de la membrane, et peut être 
enlevé presque complètement ( 95 à $100 \%$ selon Eylar et al. [46]) par l'action de la neuraminidase. On peut suivre précisément l'action de cet enzyme sur la membrane de l'hématie, en mesurant en parallèle la variation de mobilité des globules rouges, et la quantité d'acide sialique libéré dans le milieu de suspension.

A titre d'exemple d'application de notre méthode, nous rapportons les résultats électrophorétiques obtenus lors d'une étude de désialylation des hématies.

Rappelons que la mobilité électrophorétique des hématies dépend de la nature du milieu de suspension, de son $\mathrm{pH}$ et de sa force ionique. Ces paramètres doivent donc être bien déterminés et constants au cours de l'expérience. Pour être dans des conditions "physiologiques », assurant leur bonne conservation, les hématies doivent être suspendues dans un milieu isotonique (osmolalité $=0,3 \mathrm{osm} . / \mathrm{l}$ ), tamponné à $\mathrm{pH}=7,4$ (valeurs caractéristiques du plasma). Nous utilisons en général un tampon phosphate- $\mathrm{NaCl}$ de force ionique $5 \times 10^{-3} \mathrm{M}$ ou $10^{-2} \mathrm{M}$, dont l'isotonicité (osmolalité finale $~ 300 \mathrm{mosm} . /$ ) est assurée avec du saccharose [37].

5.4.1 Mobilité moyenne. - Le tableau III donne pour différents sujets la mobilité électrophorétique mesurée avant et après traitement par la neuraminidase, et la quantité d'acide sialique libéré correspondante.

Ces résultats sont reportés dans la figure 17 , qui fait correspondre pour chaque sujet la quantité d'acide sialique libéré à la variation de mobilité
Tableau III. - Relation entre la chute de mobilité érythrocytaire et la quantité d'acide sialique enlevé, pour 6 donneurs différents.

[Relation between the erythrocyte mobility decrease and the released sialic acid amount, for 6 different donors.]

\begin{tabular}{|c|c|c|c|}
\hline Sujets & $\begin{array}{c}\mu \\
10^{-4} \mathrm{~cm}^{2} \cdot \mathrm{s}^{-1} \cdot \mathrm{V}^{-1}\end{array}$ & $\begin{array}{c}\Delta \mu=\mu-\mu_{T} \\
10^{-4} \mathrm{~cm}^{2} \cdot \mathrm{s}^{-1} \cdot \mathrm{V}^{-1}\end{array}$ & $\begin{array}{c}\text { Sialique } \\
\text { libéré } \\
\mu \mathrm{g} / 10^{10} \mathrm{GR}\end{array}$ \\
\hline 1 & 2,32 & & \\
2 & 1,09 & 1,23 & 168 \\
& 2,23 & & \\
3 & 1,12 & 1,11 & 151 \\
& 2,29 & & \\
& 1,27 & 1,02 & 113 \\
4 & 1,06 & 1,23 & 151 \\
& 2,12 & & \\
5 & 1,49 & 0,63 & 45 \\
& 1,02 & 1,10 & 110 \\
6 & 2,15 & & \\
& 1,54 & 0,61 & 43 \\
& 1,23 & 0,92 & 100 \\
& 2,18 & & \\
& 0,93 & 1,25 & 200 \\
\hline
\end{tabular}

$\Delta \mu$ entre l'échantillon témoin et l'échantillon traité. On remarque que ces 2 paramètres sont parfaitement corrélés, mais de manière non linéaire, la mobilité

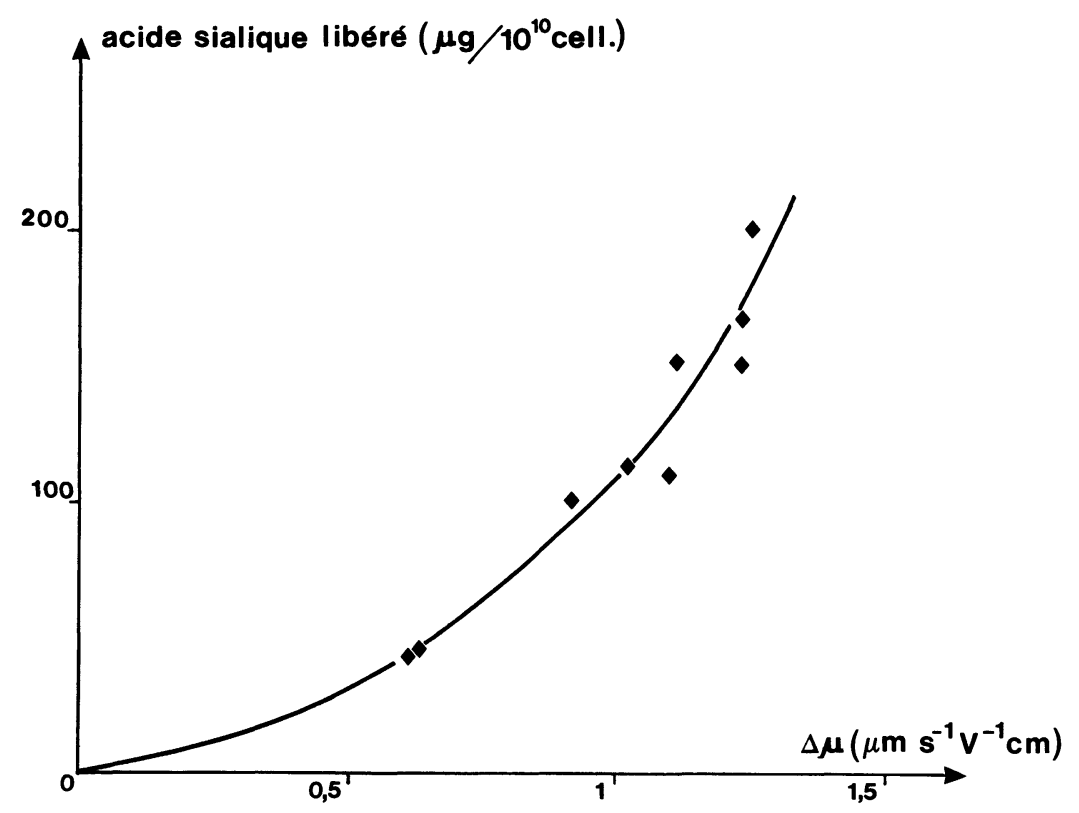

Fig. 17. - Différence de mobilité électrophorétique entre hématies témoins et hématies désialylées, représentée en fonction de la quantité d'acide sialique libéré.

[Electrophoretic mobility difference between control and desialylated erythrocytes, as a function of the released sialic acid amount.] 
variant moins vite que la quantité d'acide sialique libéré ; de plus, à désialylation maximum, la mobilité résultante est loin d'être négligeable, puisqu'elle vaut environ $40 \%$ de la mobilité témoin $\left(\Delta \mu / \mu_{\mathrm{T}} \approx\right.$ $60 \%$ dans notre exemple).

Ainsi le traitement à la neuraminidase, en libérant l'acide sialique de la membrane, fait diminuer la charge électrique superficielle donc la mobilité.

La non-linéarité entre sialique libéré et mobilité pourrait s'expliquer en partie par la non-linéarité entre densité superficielle de charge et mobilité dans la théorie classique de l'électrophorèse $[37,5]$. Rappelons que dans cette théorie, les charges électriques sont distribuées à la surface de la particule considérée comme lisse, et la mobilité est reliée à la charge «apparente » de la particule, comprenant toutes les charges situées à l'intérieur d'une surface de cisaillement (en particulier les ions adsorbés de signe contraire); cette charge «électrocinétique » est donc généralement plus petite que la charge « réelle » de la particule, trouvée par exemple par titrage. Dans la littérature, la perte de charge calculée à partir de la variation de $\mu$ est ainsi sousestimée d'un facteur 2 à 3 par rapport à la quantité d'acide sialique libéré. Une interprétation récente plus réaliste fait intervenir une distribution spatiale des charges sur une certaine épaisseur [47-49]; le modèle de la glycocalyx chargée de manière homogène laisse prévoir une variation de mobilité directement proportionnelle à la quantité de sialique libéré ; la variation observée expérimentalement, non linéaire, peut être expliquée par une modification de la structure de la glycocalyx au cours du traitement : en effet si l'on admet [47] que la réduction de charge de la glycocalyx s'accompagne d'une réduction du paramètre de frottement (ce qui entraînerait alors une augmentation de $\mu$ à densité de charge cons- tante), il s'ensuit que la mobilité diminue moins rapidement que la densité de charge.

L'existence d'une mobilité résiduelle à désialylation maximum suggère que d'autres groupements ionisés doivent participer à la charge globale négative du globule rouge. Plusieurs auteurs ont déjà observé ce fait : Luner et al. par exemple [50] trouvent une réduction de mobilité maximum de $90 \%$, à force ionique physiologique. Dans notre étude, le taux de réduction maximum de $60 \%$ est probablement dû à la faible force ionique utilisée : Donath et Lerche [47] ont observé en effet que le taux de réduction de la mobilité croît avec la force ionique du milieu de suspension, pour une même quantité de neuraminidase, et l'interprètent selon leur modèle de charge volumique, la charge résiduelle $(10 \%$, indépendamment de la force ionique) étant alors répartie à la surface de l'hématie. Par ailleurs, Levine et al. [48] ont montré qu'une mobilité résiduelle est possible même si la charge nette résultante après désialylation est nulle, dès lors que l'on suppose une distribution de charge non uniforme dans la glycocalyx...

\subsubsection{Spectres de mobilité. - La technique VRT} nous a permis de mettre en évidence une modification de la distribution des mobilités pour les hématies traitées.

La figure 18a donne le spectre de mobilité des hématies d'un échantillon témoin incubé dans le tampon, sans neuraminidase; la largeur à mi-hauteur $\Delta \mu$ de ce spectre (que l'on peut estimer par lissage) est de $0,20 \times 10^{-4} \mathrm{~cm}^{2} \cdot \mathrm{s}^{-1} \cdot \mathrm{V}^{-1}$, c'est-à-dire de $8,1 \%$ en valeur relative. La dispersion $\Delta \mu$ des valeurs de mobilité autour de la moyenne est caractéristique de la distribution de la densité de charge superficielle dans la population de globules rouges, et n'est pas un élargissement artificiel dû à l'appareil,

b)

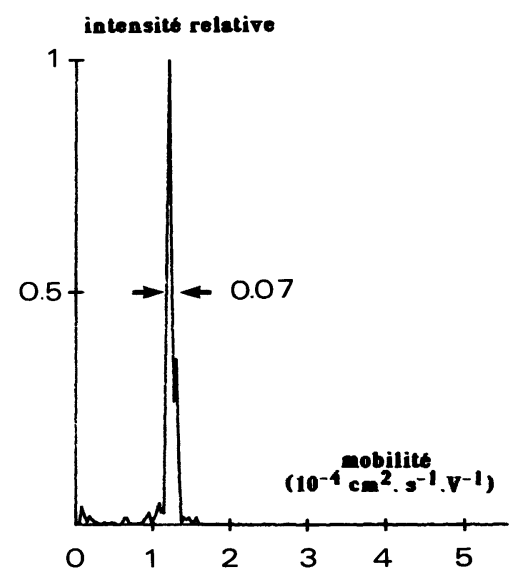

Fig. 18. - Spectres de mobilité électrophorétique d'hématies (incubées à $37^{\circ} \mathrm{C}$ ), suspendues dans un tampon phosphate- $\mathrm{NaCl} 10^{-2} \mathrm{M}$ : a) suspension d'hématies témoins, b) suspension d'hématies désialylées.

[Electrophoretic mobility spectra of erythrocytes (incubated at $37^{\circ} \mathrm{C}$ ), suspended in a $10^{-2} \mathrm{M}$ phosphate- $\mathrm{NaCl}$ buffer : a) control cells, b) desialylated cells.] 
puisque l'on obtient une résolution de $1 \%$ lorsque toutes les cellules migrent à la même vitesse [37]. La figure $18 \mathrm{~b}$ correspond à l'échantillon incubé avec la neuraminidase : celui-ci a un spectre beaucoup plus étroit $\left(\Delta \mu=0,07 \times 10^{-4} \mathrm{~cm}^{2} \cdot \mathrm{s}^{-1} \cdot \mathrm{V}^{-1}\right)$, ce qui indique que les acides sialiques sont distribués de manière plus hétérogène dans la population de cellules normales que ne le sont les charges résiduelles laissées après le traitement par la neuraminidase.

Si l'on mesure ensemble l'échantillon témoin et l'échantillon désialylé en les mélangeant dans le milieu de suspension, on observe toujours une nette différence de largeur entre les deux pics du spectre de mobilité (Fig. 19).

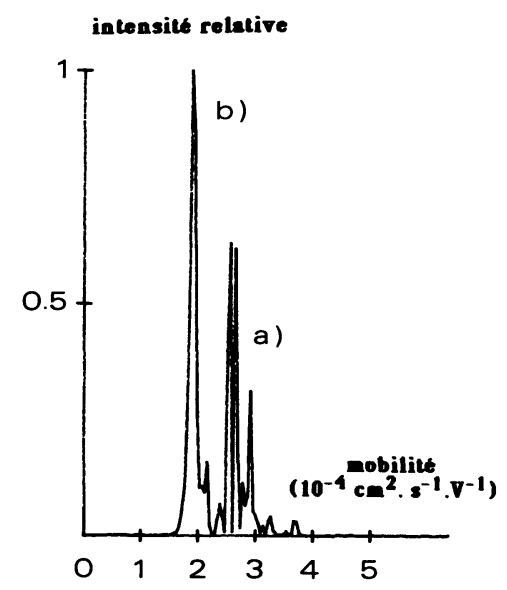

Fig. 19. - Spectre de mobilité électrophorétique d'une suspension contenant 2 types d'hématies : a) témoins, b) désialylées.

[Electrophoretic mobility spectrum of a mixture of control (a) and desialylated (b) cells.]

\section{Conclusion.}

Une nouvelle méthode de microélectrophorèse, utilisant un vélocimètre optique à réseau de transmission, a été développée. Le dispositif expérimental mis au point (système optique spécialement adapté, cuve de mesure pratique avec plans stationnaires bien définis, système de traitement de signal performant et souple d'emploi) permet d'obtenir rapide- ment des spectres de mobilité de cellules ou particules en suspension.

Nos études électrophorétiques sur les globules rouges humains $[37,51]$ confirment un certain nombre de données de la littérature, en ce qui concerne la mobilité moyenne des hématies dans des conditions physico-chimiques précises. De plus, en exploitant la précision de la méthode et son pouvoir de résolution en mobilité, nous avons pu détecter dans certains cas de faibles variations de mobilité (suivant le mode de préparation des globules rouges, leur âge in vivo, ou la nature du milieu de suspension), observer des altérations dans la distribution des mobilités (par exemple par incubation, ou lors de la désialylation), ou encore corréler la mobilité avec certaines propriétés rhéologiques de l'hématie (déformabilité, agrégation érythrocytaire).

Le vélocimètre à réseau de transmission (VRT) peut remplacer utilement les vélocimètres Dopplerlaser, à condition bien sûr que les particules étudiées soient visibles en microscopie en fond noir. En plus des avantages déjà décrits (meilleure résolution en vitesse, plus grande facilité d'emploi) [35-37], remarquons que la direction de la vitesse des particules peut être déterminée très simplement, en tournant le réseau dans son plan jusqu'à obtention de la fréquence maximum de modulation. Par ailleurs, la technique VRT peut être utilisée à différentes longueurs d'onde ; une application intéressante serait l'étude de particules fluorescentes.

Le vélocimètre à réseau de transmission a été initialement conçu pour la mesure de la mobilité électrophorétique des cellules sanguines, pour lesquelles il est parfaitement adapté. L'appareil peut être utilisé directement pour d'autres applications vélocimétriques :

- électrophorèse d'autres types de «particules » (latex, gouttes d'huile, bulles d'air, autres cellules biologiques, etc.) ;

- mesures d'écoulement, de profils de vitesse (dans des conduits transparents) ;

- mesure de vitesses de sédimentation (avec un réseau horizontal);

- étude de mouvements actifs cellulaires (motilité des spermatozoïdes, des bactéries).

\section{Bibliographie}

[1] James A. M., Prog. Biophys. Biophys. Chem. 8 (1957) 95.

[2] Brinton C. C. and LAuffer M. A., Electrophoresis, Ed. M. Bier, Chapter 10 (Academic Press, New York) 1959.

[3] Seaman G. V. F., The red blood cell, Ed. Mac N. Surgenor D. (Academic Press, New York) Vol. 2, $2^{\text {nd }}$ ed. (1975) pp. 1135-1229.
[4] JAMES A. M., Surface and Colloid Science, Eds. R. J. Good and R. R. Stromberg (Plenum Press, New York and London) Vol. 11 (1979) pp. 121185.

[5] HuNTER R. J., Zeta potential in colloid science, Eds. R. H. Ottewill and R. L. Rowell (Academic Press, London and New York) 1981.

[6] Delatour E., Thèse de Doctorat de $3^{\text {c }}$ Cycle (Université Paris 6) 1975. 
[7] Delatour E. and Hanss M., Rev. Sci. Instrum. 47 (1976) 1531.

[8] Delatour E. et Hanss M., J. Fr. Biophys. Méd. Nucl. 4 (1977) 173.

[9] Hakim B. H., Delatour E. and Hanss M., Rev. Sci. Instrum. 56 (1985) 462.

[10] Brooks D. E., Seaman G. V. F., Olson G. B. and BARTELS P. H., Cell Electrophoresis : Clinical Application and Methodology, I.N.S.E.R.M. Symposium $\mathrm{n}^{\circ} 11$ (North-Holland, Amsterdam) 1979, p. 409.

[11] Callan R., Johnson M., Jones R., Bater A. J., PRITCHARD J. A. V., SMITH J. and SUTHERLAND W. H., Cell Electrophoresis : Clinical Application and Methodology, I.N.S.E.R.M. Symposium $\mathrm{n}^{\circ} 11$ (North-Holland, Amsterdam) 1979, p. 431.

[12] UNGER R., SCHOEPPE G., SCHUETT W. and JeNSSEN H. L., Cell Electrophoresis : Clinical Application and Methodology, I.N.S.E.R.M. Symposium $n^{\circ} 11$ (North-Holland, Amsterdam) 1979, p. 399.

[13] Yeh Y. and Cummins H. Z., Appl. Phys. Lett. 4 (1964) 176.

[14] Drain L. E., The laser Doppler technique (Wiley J. \& sons Ltd, New York) 1980.

[15] Ware B. R. and Flygare W. H., Chem. Phys. Lett. 12 (1971) 81.

[16] Ware B. R. and Flygare W. H., J. Colloid. Interface Sci. 39 (1972) 670.

[17] UzGiris E. E., Opt. Commun. 6 (1972) 55.

[18] Uzgiris E. E., Rev. Sci. Instrum. 45 (1974) 74.

[19] Josefowicz J. and Hallett F. R., Appl. Opt. 14 (1975) 740.

[20] HaAs D. D. and Ware B. R., Anal. Biochem. 74 (1976) 175.

[21] HaRvey J. D., Walls D. F. and Woolford M. W., Opt. Commun. 18 (1976) 367.

[22] Mohan R., Steiner R. and Kaufmann R., Anal. Biochem. 70 (1976) 506.

[23] Magdalenat H., Turo P., Tivant P., Chemla M., Menez R. and Drifford M., J. Chem. Educ. 55 (1978) 12.

[24] Kaufmann R., Steiner R. and Hoffman W., Cell Electrophoresis : Clinical Application and Methodology, I.N.S.E.R.M. Symposium $n^{\circ} 11$ (North-Holland, Amsterdam) 1979, p. 435.

[25] Drifford M., Menez R., Tivant P., Nectoux P. et Dalbiez J. P., Revue Phys. Appl. 16 (1981) 19.

[26] Mahler E., Martin D., Duvivier C., Volochine B. and Stoltz J. F., Biorheology 19 (1982) 647.

[27] Dalbiez J. P., TABTI K., Derian P. J. et DRIFFord M., Revue Phys. Appl. 22 (1987) 1013.
[28] Uzgiris E. E., Prog. Surf. Sci. 10 (1981) 53.

[29] Uzgiris E. E., Adv. Colloid Interface Sci. 14 (1981) 75.

[30] WARE B. R. and HAAS D. D., Fast Methods in Physical Biochemistry and Cell Biology, Eds. R. Sha'Afi and S. Fernandez (Elsevier, Amsterdam) 1982, p. 173

[31] Ballik E. A. and Chan J. H. C., Appl. Opt. 12 (1973) 2607

[32] Berge P. et Dubois M., Brevet C.E.A. n 73-04-708 (1973).

[33] Dubois-Gance M., Thèse de Doctorat d'Etat (Univ. Paris 6) 1976.

[34] Gaster M., J. Fluid. Mech. 20 (1964) 183.

[35] Delatour E., Innov. Technol. Biol. Méd. 4 n sp. 1 (1983) 214.

[36] Delatour E. and Hanss M., Rev. Sci. Instrum. 55 (1984) 508.

[37] Delatour-Hanss E., Thèse de Doctorat d'Etat (Université Paris 13) 1988.

[38] Champeney D. C., Fourier transforms and their physical applications (Academic Press, London and New York) 1973.

[39] Mie G., Ann. Phys. 25 (1908) 377.

[40] Dukhin S. S. and DerJaguin B. V., Surface and Colloid Science, Ed. E. Matijevic (Wiley J., New York) Vol. 7 (1974).

[41] Komagata S., Res. Electrotechn. Lab. Jpn 348 (1933) 5.

[42] Van Oss C. J., Fike R. M., Good R. J. and ReINIG J. M., Anal. Biochem. 60 (1974) 242.

[43] Nordt F. J., Knox R. J. and Seaman G. V. F., Hydrogels for Medical and Related Applications, A.C.S. Symposium Series $\mathrm{n}^{\circ} 31$, Ed. J. D. Andrade (Washington) 1976, p. 225.

[44] SMrth B. A. and WARE B. R., Contemporary Topics in Analytical and Clinical Chemistry, Eds. D. N. Hercules, G. M. Hieftje, L. R. Snyder and M. A. Evenson (Plenum Press, New York) Vol. 2 (1978) p. 29.

[45] Koutsouris D. et Hanss M., Innov. Technol. Tech. Biol. Méd. 1 (1983) 85.

[46] Eylar E. H., Madoff M. A., Brody O. V. and OnCley J. L., J. Biol. Chem. 237 (1962) 1992.

[47] Donath E. and Lerche D., Bioelectrochem. Bioenerg. 7 (1980) 41.

[48] Levine S., Levine M., SharP K. A. and-Brooks D. E., Biophys. J. 42 (1983) 127.

[49] Snabre P. and Mills P. Colloid Polym. Sci. 263 (1985) 494.

[50] Luner S. J., Sturgeon P., SzKLarek D. and Mc Quiston D. T., Vox Sang. 28 (1975) 184.

[51] Delatour-Hanss E., à paraître. 